\title{
Quantum information processing, operational quantum logic, convexity, and the foundations of physics
}

\author{
Los Alamos Technical Report LA-UR 03-1199
}

Howard Barnum * a

${ }^{a}$ CCS-3: Modelling, Algorithms, and Informatics. Mail Stop B256

Los Alamos National Laboratory, Los Alamos, NM 87545 USA. barnum@lanl.gov

Quantum information science is a source of task-related axioms whose consequences can be explored in general settings encompassing quantum mechanics, classical theory, and more. Quantum states are compendia of probabilities for the outcomes of possible operations we may perform a system: "operational states." I discuss general frameworks for "operational theories" (sets of possible operational states of a system), in which convexity plays key role. The main technical content of the paper is in a theorem that any such theory naturally gives rise to a "weak effect algebra" when outcomes having the same probability in all states are identified, and in the introduction of a notion of "operation algebra" that also takes account of sequential and conditional operations. Such frameworks are appropriate for investigating what things look like from an "inside view," i.e. for describing perspectival information that one subsystem of the world can have about another. Understanding how such views can combine, and whether an overall "geometric" picture ("outside view") coordinating them all can be had, even if this picture is very different in structure from the perspectives within it, is the key to whether we may be able to achieve a unified, "objective" physical view in which quantum mechanics is the appropriate description for certain perspectives, or whether quantum mechanics is truly telling us we must go beyond this "geometric" conception of physics.

Keywords: quantum information ; foundations of quantum mechanics ; quantum computation ; quantum logic ; convexity ; operational theories PACS codes:

\section{Introduction}

The central question quantum mechanics raises for the foundations of physics is whether the attempt to get a physical picture, from "outside" the observer, of the observer's

\footnotetext{
*Thanks to the US DOE for financial support.
} 
interaction with the world, a picture which views the observer as part of a reality which is at least roughly described by some mathematical structure, which is interpreted by pointing out where in this structure we, the observers and experimenters, show up, and why things end up looking as they do to observers in our position, is doomed. The "relative state" picture that arises when one tries to describe the whole shebang by an objectively existing quantum state is unattractive, and many seek to interpret quantum states instead as subjective, "information" about how our manipulations of the world could turn out. Whatever else they may be the quantum states of systems clearly are compendia of probabilities for the outcomes of possible operations we may perform on the systems: "operational states." An operational theory is a specification of the set of possible operations on a system and a set of admissible operational states. This "operational" point of view can be useful whether one wants to consider the operational theory as for some reason all we can hope for, or as a description of how perspectives look within an overarching theory such as the relative state interpretation (RSI).

While it has not yet made a decisive contribution toward resolving this tension, by focussing on the role of information held (through entanglement or correlation) or obtained (by measurement) by one system about another QIP concentrates one's attention on the practical importance of such measurements, and develops flexibility in moving between the inside and outside views of such information-gathering processes. It thus provides tools and concepts, as well as the ever-present awareness, likely to be useful in resolving this tension, if that is possible.

This paper is dedicated to the memories of two researchers in quantum foundations, who I knew only through their collaborators and their work: Rob Clifton and Gottfried T. ("Freddy") Rüttiman. They will continue to influence and inspire for the duration of the intellectual adventure of understanding, at the deepest level, our theories of the world. Their work is particularly relevant to the themes of this paper. Algebraic quantum field theory is an example of integrating local perspectives (local *-algebras of observables) into a coherent overall structure; Clifton made deep investigations into foundational issues in AQFT - for example, Clifton and Halvorson (2001) considers entanglement in this setting. He was also involved in one of the most spectacular successes to date of the project of applying quantum information-theoretic axioms to quantum foundations (Clifton et al., 2002). Rüttiman's work involved, for example, linearization theorems for lattice-based quantum logics (Rüttiman, 1993) which parallel and prefigure the ones discussed herein for convex effect-algebras, and investigation of the relation between the property lattice and face lattice of a state space (Rüttiman, 1981).

The paper is organized as follows. Section 2 considers some salient general implications of QIS for foundational questions (irrespective of its contributions to this project). Section 3 discusses the relative state and "subjective" views on the foundations of quantum mechanics. Section 4 discusses whether and how the perspectives of different observers can be combined, via tensor products and other constructions. Section [5] constructs "weak effect algebras" from probability compendia via identification of probabilistically equivalent outcomes, reviews operational quantum logic, especially convex effect algebras, and 
introduces the notion of operation algebra which formalizes the notion of doing operations in sequence, possibly conditioned on the results of previous operations. In Section 7. I briefly consider uses of the framework in applying QIP ideas to foundational questions.

A major part of empirical quantum logic is "deriving quantum mechanics." The hope is that if this can be done with axioms whose operational, information-processing, or information-theoretic meaning is clear, then one will have a particularly nice kind of answer to the question "Why quantum mechanics?" QI/QC provides a source of axioms, with natural interpretations involving the possibility or impossibility of informationtheoretic tasks. This is likely to contribute to whichever mode of resolution turns out to be right. Within the "geometric" or "objective overall picture" resolution, one might obtain the answer: Why quantum mechanics? "Because it's the sort of structure you'd expect for describing certain perspectives (of the sort beings like us wind up with) that occur "from the inside point of view" within an overarching picture of this [fill in the blank] sort." The blank might be filled in with a specific overarching physical theory, or with fairly general features. A similar answer might arise from the more "subjectivist" point of view on quantum states. Why quantum mechanics? "Because it's the sort of structure you'd expect for describing the perspectives "from the inside point of view" within a reality of this sort, which reality is however not completely describable in physical terms, so that these perspectives are as good as physics ever gets." Those who anticipate or hope for a physical picture, including relative state-ers, and those who think such an overarching physical picture unlikely to emerge, can nevertheless fruitfully pursue similar projects using axiomatic arguments involving the notion of "operational theory" to derive quantum mechanics, to understand, how it differs from or is similar to other conceivable theories, and the extent to which it does or does not follow from elementary conceptual requirements (one way in which it could be "a law of thought") or, in a more Kantian or perhaps "anthropic" way, from the possibility of rational beings like us (a different way in which it could be "a law of thought"). Details might depend on one's orientation: subjectivists might be more inclined to axioms stressing the formal analogies between density matrices and probability distributions, and between quantum "collapse" and Bayesian updating of probability distributions (Fuchs, 2001a). But since on the "overarching physical picture with perspectives" view the probabilities are also tied to a "subjective," perspectival element, the Bayesian analogy is quite natural on this picture too. The close link between "empirical operational theories" and perspectival information that one subsystem of the world can have about another, and the importance of tasks, of what can and cannot be done from a given perspective, suggests that generalized information theory and information processing, of which QIS supplies a main example, will play a major role in this project.

\section{QIP: The power of the peculiar}

Virtually all of the main aspects of quantum mechanics exploited in QIP protocols have been understood for decades to be important peculiarities of quantum mechanics. The nonlocal correlations allowed by entanglement are exploited by better-than-classical com- 
munication complexity protocols (Buhrman et al., 1997); the necessity of disturbance when information is gathered on a genuinely quantum ensemble (Fuchs and Peres, 1995; Barnum, 1998, 2001; Banaszek, 2001; Bennett et al., 1994; Barnum et al., 2001), closely related to the "no-cloning theorem" (Wootters and Zurek, 1982) and no-broadcasting theorem (Barnum et al., 1996; Lindblad, 1999), is the basis of quantum cryptography; the ability to obtain information complementary to that available in the standard computational basis is the heart of the historic series of algorithms due to Deutsch (1985), Deutsch and Jozsa (1992), Simon (1997), Bernstein and Vazirani (1997), and culminating in Shor's (1994; 1997) polynomial-time factoring algorithm. These peculiarities are no longer just curiosities, paradoxes, philosophers' conundrums, they now have worldly power.

A number of more specific and/or technical points on which QIP has contributed, or shows potential to contribute, something new to old debates can be identified. First, QIP provides tools with which to analyze much more precisely and algorithmically questions of what can and cannot be measured (Wigner, 1952; Araki and Yanase, 1960; Reck et al., 1994), or otherwised accomplished, either precisely or approximately, in quantum mechanics. Some measurements are even uncomputable in essentially the same sense as are some partial recursive functions in classical computer science. This raises the issue of the extent to which "operational" limitations, including basic and highly theoretical ones such as computability, should be built into our basic formalisms, and what it means for the interpretation of those formalisms and the "reality" of the objects they refer to, if they are not. Second, QIP techniques and concepts such as error-correction and active and passive stabilization and control promise to allow a much more systematic approach than previously to experiments and thought-experiments suggested by foundational investigations. Third, QIP has demonstrated the power of taking the formal analogy between quantum density matrices and classical probability distributions seriously. Most things one does with probability distributions in classical information theory have (sometimes multiple) natural quantum analogues when quantum states replace probability distributions. Fourth, QIP provides a source of natural "operational" questions about whether certain information-processing tasks can or cannot be performed, usable when considering empirical theories more general than quantum mechanics. Also, QIP may be a natural source of examples of empirical theories. These arise when one considers attempts to perform quantum information processing with the restricted meansavailable in some proposed implementation of quantum computing. For example, QIP considerations stimulated some of us (Barnum et al., 2002) to generalize the notion of "entanglement" to pairs of lie algebras and beyond that to pairs of ordered linear spaces.

\section{Relative state vs. information interpretation of quantum mechanics}

The central tension in interpreting quantum mechanics is between the idea that we are part of a quantum world, made of quantum stuff interacting with quantum stuff, evolving according to the Schrödinger equation, and the apparent fact that when we evolve so as to correlate our state with that of some other quantum system which is initially in 
a superposition, we get a single measurement outcome, with probabilities given by the squared moduli of coefficients of the projections of the state onto subspaces in which we see a definite measurement outcome. The RSI reconciles these ideas by taking the view that the experience of obtaining a definite measurement result is how things appear from one point of view, our subspace of the world's Hilbert space, and the full state of the world is indeed a superposition. As I see it the correct way, on this view, to account for the appearance that there is a single measurement result, is the idea that the experience of a conscious history is associated with definite measurement results, so that consciousness forks when a quantum measurement is made (Barnum, 1990). Just as there is no consciousness whose experience is that of the spacetime region occupied by you, me, Halley's comet, and the left half of Georges Sand, so, after a measurement has correlated me with the the $\mathrm{z}$-spin of an initially $\mathrm{x}$-polarized photon, there is no consciousness whose experience is that of the full superposition (or, once these branches of me are decohered, of the corresponding mixture). Understanding why this happens as it does would appear to involve psychological/philosophical considerations about how minds are individuated. A more precise account must await a better scientific understanding of consciousness, though there are probably some useful things to be said by philosophers, psychologists, biologists, and decoherence theorists. It is deeply bound up with the problem of choosing a "preferred basis" in the relative state interpretation (i.e., the question, "relative to what?"), and also with the problem of what tensor factorization of Hilbert space to choose in relativizing states, which appears in this light as the question of which subsystems of the universe support consciousness. The stability of phenomena and their relations enforced by decoherence may underly the ability to support consciousness.

Despite sometimes conceding when pressed that they can't show the RSI is inconsistent, its opponents also sometimes claim it is inconsistent for an observer to view him or herself as described by quantum theory (Fuchs and Peres, 2000). I am not aware of a rigorous argument for this, though. Even an argument within a toy model would be valuable. But ven if it is shown that it would be inconsistent for an observer herself to have a complete quantum-mechanical description of herself, the system she is measuring, and the part of the universe that decoheres her "in the pointer basis," that does not show that such a description is itself inconsistent. Similar "bizarre self-referential logical paradoxes" Fuchs and Peres (2000) seem just as threatening (or not) for a classical description.

Some Bilodeau (1996) think that QM is telling us we must abandon the "geometric" conception of physics as giving us an "outside view" of reality. But I think that rather than just welcoming the ability to view quantum mechanics as only appropriate to describing an observer's perspective on a system, revelling in the subjectivity of it all, the way it perhaps leaves room for mind, freewill, etc... as unanalyzed primitives, it is still promising to try to get a grip on these matters "from an outside point of view." An analogy might be special relativity. Here, an overarching picture was achieved by taking seriously the fact that position and time measurements are done via operations, from the perspective of particular observers. The heart of the theory is to coordinate those perspectives into a global Minkowski space structure, explaining in the process certain aspects of the local operational picture (like restrictions on the values of velocity measurements). I don't 
think that we should yet give up on an attempt at such coordination in the quantum case, perhaps celebrating the supposed fact that quantum mechanics has shown us that it will be impossible to achieve under the aegis of physics.

An important point brought out by the attempt at a relative state interpretation of quantum mechanics is the need to bring in, in addition to Hilbert space, notions of preferred subsystems ("experimenter" and "system" perhaps also the "rest of the world") or preferred orthogonal subspace decompositions (choice of "pointer basis" (Zurek, 1981)). It seems unlikely, as Benjamin Schumacher likes to point out, that a Hilbert space, Hamiltonian, and initial state, will single out preferred subspace decompositions in which dynamics looks nontrivial, hence the RSI should involve aspects of physics beyond Hilbert space. Schumacher also points out that a Hamiltonian evolution on a Hilbert space can be made to look trivial by a time-dependent change of basis. If one takes the view that "the classical world" is supposed to emerge from this structure (Hilbert space, Hamiltonian, and initial state), then perhaps such transformations are legitimate. On the other hand, they are not wholly trivial: if one specifies a Hamiltonian dynamics on a Hilbert space, one is implicitly specifying two groups of canonical isomorphisms between a continuum of Hilbert spaces, continuously parametrized by time. One of them says what we mean by "same Hilbert space at different times," providing a framework with respect to which we can then define a Hamiltonian evolution specified by the other one. If we could pick out a set of subspaces that are special with respect to this structure, that would be interesting. I have doubts that we can; I also like Schumacher's criticism that this specification of "two connections on a fiber bundle instead of just one" seems mathematically unnatural. But I am not wholly convinced by Schumacher's criticisms. I view the RSI less as a way of getting the classical world emerge from Hilbert space, and more as a way of giving a realistic interpretation to Hilbert space structure in the presence of additional structure such as preferred bases or subsystem decompositions that represent other aspects of physics. Schumacher views his arguments as showing that one needs these additional aspects of physics - "handles on Hilbert space" - to get a canonical identification of, say, bases from one time to the next (say the spin-up/down basis in a given reference frame). He interprets this as showing the appropriateness of Hilbert space descriptions for subsystems where the special structure lies in relations to other systems (such as measuring appartus), and the inappropriateness of the Hilbert space structure for the description of the whole universe. There are plenty of such non-Hilbert space aspects of physics, involving symmetries, spacetime structure. The need for renormalization and the difficulties with quantum gravity suggest some difficulty in squaring quantum mechanics with some of these "geometrical," "outside" aspects of physics. Perhaps the distasteful aspects of the quantum-mechanical outside view may vanish once such a squaring, with whatever flexing is necessary from both sides, is accomplished.

Bell showed that nonlocal hidden variables are the only non-conspiratorial way to realistically model the statistics of quantum measurements. (Non-conspiratorial refers to a prohibition on explaining the statistics of quantum measurements by correlations between the hidden variables and what we "choose" to measure.) But when we are contemplating quantizing the spacetime metric or otherwise unifying gravity and quantum mechanics, 
perhaps it is not too farfetched to imagine that spacetime and causality will turn out to be emergent from a theory describing a structure at a much deeper level....if this structure contains things whose effects, at the emergent level of spacetime, can be interpreted as those of "nonlocal hidden variables," this should hardly surprise or dismay us.

My view toward the RSI with macroscopic superpositions is much like Einstein's toward taking quantum mechanics as a complete physical theory: I just don't think the universe is like that. Schulman (1997) proposes to retain essentially a one-Hilbert space, statevector evolving according to the Schrodinger equation, no-collapse version of quantum mechanics, interpreted realistically, but to bring in cosmology and statistical mechanics and argue that symmetric consideration of final conditions along with the usual initial conditions (that the universe was once much denser and hotter than it is now) rules out macroscopic superpositions. There is a lot to do to make this persuasive. It is certainly an ingenious and appealing idea. And if it does work, I am fairly happy to retain the rest of the relative state metaphysics, now that I will not be committed to the disturbing existence of forking Döppelgangers in subspaces of Hilbert space decohered from me.

\section{The combination of perspectives}

We should continue to investigate both the inside and outside views of quantum systems, and in interpretational matters to pursue a better understanding both of the possibility of viewing quantum theory as about the dynamics of information-like, perhaps subjective, states, and of the possibility of viewing it as about the sorts of entanglement and correlation relations that can arise between systems. A prime example of a worthwhile program along the former lines is the Caves-Fuchs-Schack Bayesian approach; a prime example of a worthwhile program along the latter lines is understanding how the probabilities for collapse can be understood within the RSI Deutsch (1999); Wallace (2002), also as something like a Bayesian process of "gaining more information about which branch of the wavefunction we are in." The similarity between these two programs is an example of how the operational approach is relevant to both: investigate quantum mechanics' properties as a theory of perspectives of subsystems on other systems, without prejudging whether or not the perspectives will turn out to be coordinatable into an overarching picture -indeed, while trying to ferret out how this might happen or be shown to be inconsistent, and how this possibility or impossibility may be reflected in the operational, perspective-bound structures.

The Rovelli-Smolin "relational quantum mechanics" approach suggests ways in which quantum mechanics could be good for describing things from the point of view of subsystems, but not appropriate for the entire universe, but in which nevertheless there exists a mathematical structure - something like a topological quantum field theory (TQFT) or spin foam - in which these local subsytem points of view are coordinated into an overall mathematical structure which, while its terms may be radically different from those we are used to, may still be viewed as in some sense "objective." It is still far from clear that this can allow us to avoid the more grotesque aspects (proliferating macroscopic superpo- 
sitions viewed as objectively existing) and remaining conceptual issues (how to identify a preferred tensor factorization, and/or preferred bases, in which to identify "relative states") of the Everett interpretation.

In TQFT's or spin networks and generalizations, the description appropriate to "perspectives" is still Hilbert spaces, but only in special cases do these combine as tensor products. If we view a manifold as divided into "system" and "observer" via a cobordism, then as the "observer" gets small enough, while the "system" gets larger, we start getting, not the increase in Hilbert-space size to describe the system that we might expect as the system gets larger, but a decrease in Hilbert-space size whose heuristic interpretation might be that the observer has gotten so small that it no longer has the possibility of measuring all the operators needed to describe the "large" Hilbert space one might have expected. The Hilbert space does not describe the "large" rest of the world; it describes the relation between a small observer and the larger rest of the world.

In these theories, we might see how the quantum description of certain perspectives could arise as a limiting case of some more general type of perspective, which necessarily also arises in an overarching structure that includes quantum-mechanical perspectives in a physically reasonable way. Or we might see how a non-tensor product law of combination of subsystems - quantum or not - could be relevant in some situations. This is just the sort of thing that operational quantum logic aspires to investigate, and that might be related to the ability to perform, or not, information-processing tasks.

\section{Frameworks for empirical operational theories}

In this section I will introduce frameworks I find particularly useful for thinking about empirical operational theories. David Foulis (1998) has provided a good review of the general area of mathematical descriptions of operational theories (which he calls "mathematical metascience"). That review stresses concepts similar to those I use here, notably that of effect algebras," introduced under this name by Foulis and Bennett (Foulis and Bennett, 1994), but also, as "weak orthoalgebras" in Greuling (1989), and independently, in an order-theoretic formulation, as "difference posets" (D-posets, for short) by Kôpka and Chovanec (1994). Longer and more technical introductions are available in Foulis (2000) and Wilce (2000).

\subsection{Probabilistic equivalence}

My preferred approach to operational theories starts from the compendia of probabilities, that are empirically found to be possible for the different results of different possible operations on a system, and constructs various more abstract structures for representing aspects of empirical theories - effect algebras, classical probability event-spaces, $C^{*}$-algebraic representations, spaces of density operators on Hilbert spaces, orthomodular lattices, or what have you - from these. With most such types of abstract structures, the possibility

of constructing them from phenomenological theories (sets of compendia of probabilities 
for measurement outcomes) will impose restrictions on these sets of compendia, and the nature of these restrictions constitutes the empirical significance of the statement that our empirical theory has this abstract structure. This approach promises to systematize our understanding of a wide range of empirical structures and their relationships, both mathematically and in their empirical significance. The relationship to the probabilities of experimental outcomes has always been a critical part of understanding these structures as empirical theories. The space of "states" on such structures is also often a crucial aid to understanding their abstract mathematical structure. This is of a piece with the situation in many categories of mathematical objects. $[0,1]$ is a particularly simple example of many categories of "empirical structure," and a state is a morphism onto it; understanding the structure of some more complex object in the category in terms of the set of all morphisms onto this simple object is similar to, say, understanding the structure of a group in terms of its characters (morphisms to a particularly simple group).

In this project I make use of an idea which has come in for a fair amount of criticism, but has been with us from early in the game (cf. e.g. (Mackev, 1963), Cooke and Hilgevoord (1981) (who even ascribe it to Bohr), Ludwig (1983a), Mielnik (1969) p. 14). This is the notion of "probabilistic equivalence": two outcomes, of different operational procedures, are viewed as equivalent, if they have the same probability "no matter how the system is prepared," i.e., in all admissible states of the phenomenological theory. An interpretation of equivalent outcomes as "exhibiting the same effect of system on apparatus" is probably due to Ludwig, perhaps motivating his term "effect" for these equivalence classes (at least in the quantum case). It helps forestall the objection that two outcomes equivalent in this sense may lead to different probabilities (conditional on the outcomes) for the results of further measurements. They are equivalent only as concerns the effect of the system on the apparatus and observer, not vice versa. The criticism implicitly supposes a framework in which operations may be performed one after the other, so that outcomes of such a sequence of $N$ measurements are strings of outcomes $a_{1} a_{2} \ldots a_{N}$ of individual measurements. Then a stricter notion of probabilistic equivalence may be introduced, according to which two outcomes $x$ and $y$ are equivalent if for every outcome $a, b$ the probability of $a x b$ is the same as that of $a y b$, in every state.

Before considering in detail the derivation of the structure of the set of probabilistic equivalence classes ("effects") of an operational theory, I will introduce some of the abstract structures we will end up with: effect algebras and "weak effect algebras," motivating them (in the case of effect algebras) with classical and quantum examples.

Definition 1 An effect algebra is an object $\langle\mathcal{E}, 1, \oplus\rangle$, where $\mathcal{E}$ is a set of "effects, " $1 \in \mathcal{E}$, and $\oplus$ is a partial binary operation on $\mathcal{E}$ which is (EA1) strongly commutative and (EA2) strongly associative. The qualifier "strongly," which is not redundant only because $\oplus$ is partial, indicates that if the sums on one side of the equations for commutativity and associativity exist, so do those on the other side, and they are equal. In addition, (EA3) $\forall e \in \mathcal{E}, \exists ! f \in \mathcal{E} \quad(e \oplus f=u)$. (The exclamation point indicates uniqueness. We give this unique $f$ the name $e^{\prime}$; it is also called the orthosupplement of e.) (EA4) a $\oplus 1$ is defined only for $a=1^{\prime}$. (We will often call $1^{\prime}$ by the name " 0 ".) 
If we only require that the equalities specifying associativity $(a \oplus(b \oplus c)=(a \oplus b) \oplus c)$ and commutativity $(a \oplus b=b \oplus a)$ hold when both sides are defined, allowing the possibility that one is defined while the other is not, we call these "weak commutativity" and "weak associativity."

In the effect algebra $\mathcal{E}(\mathcal{H})$ of quantum mechanics (on a finite-dimensional Hilbert space $\mathcal{H}$, say), $\mathcal{E}$ is the unit interval of operators $e$ such that $0 \leq e \leq I$ on the Hilbert space, $\oplus$ is ordinary addition of operators restricted to this interval (thus $e \oplus f$ is undefined when $e+f>I), 1$ is the identity operator $I$, and $e^{\prime}=I-e$, so 0 is the zero operator. A classical example is the set $\mathcal{F}$ of "fuzzy sets" on a finite set $\Lambda=\left\{\lambda_{1}, \ldots, \lambda_{d}\right\}$ (which are functions from $\Lambda$ to $[0,1])$, with $\oplus$ as ordinary pointwise addition of functions (i.e. defining $f+g$ by $(f+g)(x)=f(x)+g(x)$ except that $f \oplus g$ is undefined when $f+g$ 's range is not contained in $[0,1])$, and 1 the constant function whose value is $1 .\langle\mathcal{F}, 1, \oplus\rangle$ is an effect algebra obviously isomorphic to the restriction of the quantum effect algebra on a $d$-dimensional Hilbert space to effects which are all diagonalizable in the same basis. These "fuzzy sets" may be interpreted as the outcomes of "fuzzy measurements" in a situation where there are $d$ underlying potential atomic "sharp" measurement results or "finegrained outcomes," but our apparatus may have arbitrarily many possible meter readings, connected to these "atomic outcomes" by a noisy channel (stochastic matrix of transition probabilities, which are in fact the $d$ values taken by the function (effect) representing a (not necessarily atomic) "outcome".).

We consider various modifications of the effect algebra notion. We introduce "weak effect algebras" which are EA's in which strong associativity (EA2) is replaced by weak associativity. An orthoalgebra instead adds the axiom (OA5) that $x \oplus x$ exists only for $x=0$. The projectors on a quantum-mechanical system, with the same definitions of $1, \oplus$ as apply to more general POVM elements, are an example (as well as being a sub-effect algebra of $\mathcal{E}(\mathcal{H})$ ). Wilce considered "partial abelian semigroups," (PASes) which require only (EA1) and (EA2); various combinations of additional requirements then give a remarkably wide variety of algebraic structures that have been considered in operational quantum logic, including effect algebras, test spaces, E-test spaces, and other things. In particular, an effect algebra is a positive, unital, cancellative, PAS (see below).

A state $\omega$ on a weak effect algebra $\langle\mathcal{E}, \oplus, 1\rangle$ is a function from $\mathcal{E}$ to $[0,1]$ satisfying: $\omega(a \oplus b)=\omega(a)+\omega(b), \omega(1)=1$. A finite resolution of unity in a weak effect algebra (to be interpreted as the abstract analogue of a measurement) is a set $R$ such that $\oplus_{a \in R} a=1$.

So for a resolution of unity $R, \sum_{a \in R} \omega(a)=1$ : the probabilities of measurement results add to one. A morphism from one WEA $\mathcal{E}$ to another $\mathcal{F}$ is a function $\phi: \mathcal{E} \rightarrow \mathcal{F}$ such that $\phi(a \oplus b)=\phi(a) \oplus \phi(b)$; it is called faithful if in addition, $\phi\left(1_{\mathcal{E}}\right)=1_{\mathcal{F}}$, where $1_{\mathcal{E}}$ and $1_{\mathcal{F}}$ are the units of $\mathcal{E}$ and $\mathcal{F}$. $[0,1]$, with $\oplus$ addition restricted to the interval, is an effect algebra, so a state on $\mathcal{E}$ is a faithful morphism from $\mathcal{E}$.

I will attempt to avoid issues involving effect algebras and WEA's where $\mathcal{E}$ is infinite and infinite resolutions of unity are defined, though finite dimensional quantum mechanics is properly done that way. ((Feldman and Wilce, 1993), Bugaiski et al. (2000) and 
Gudder and Greechie (2000), for example treat these issues.) To this end I will assume that EA's and WEA's are locally finite: resolutions of unity in them have finite cardinality. For finite $d$-dimensional quantum mechanics, most things should work the same if we restrict ourselves to work with resolutions of unity into $d^{2}$ elements.

Now, I will relate this abstract structure to phenomenological theories, by showing that one can derive a natural weak effect algebra from any phenomenological theory. The operation $\oplus$ of the weak effect algebra will be the image, under our construction, of the binary relations OR $(\mathrm{V})$ in the standard propositional logics (one for each measurement) of propositions about the outcomes of a given measurement. (This is one justification for calling effect algebras "logics".)

In order to describe this construction, we first review Boolean algebras. A Boolean algebra is an orthocomplemented distributive lattice. A lattice is a structure $\langle L, \vee, \wedge\rangle$, where $L$ is a set, $\vee, \wedge$ total binary operations on $L$ with the following properties. Both operations are associative, commutative, and idempotent (idempotent means, e.g., $(a \wedge a=a)$ ). In addition, together they are absorptive: $a \wedge(a \vee b)=a, a \vee(a \wedge b)=a$. $\vee$ is usually called join, $\wedge$ is usually called meet. These properties are satisfied by letting $L$ be any powerset (the set of subsets of a given set), and the operations $\vee, \wedge$ correspond to $\cup, \cap$. For $L=2^{X}$ (the power set of $X$ ) we call this lattice the subset lattice of $X$. An important alternative characterization of a lattice is as a set partially ordered by a relation we will call $\leq$. If every pair $(x, y)$ of elements have both a greatest lower bound (inf) and a least upper bound (sup) according to this ordering, we call these $x \wedge y$ and $x \vee y$, respectively, and the set is a lattice with respect to these operations. Also, for any lattice as defined above, we may define a partial ordering $\leq$ such that $\wedge, \vee$ are inf, sup, respectively, in the ordering. So the two characterizations are equivalent.

A lattice is said to be distributive if meet distributes over join: $a \vee(b \wedge c)=(a \vee b) \wedge(a \vee c)$. (This statement is equivalent to its dual (the statement with $\wedge \leftrightarrow \vee$ ).) If $L$ contains top and bottom elements with respect to $\leq$, we call them 1 and 0 . They may be equivalently be defined via $a=a \wedge 1, a=a \vee 0$ for all $a \in L$. We define $b$ to be a complement of $a$ if $a \wedge b=0$ and $a \vee b=1$. Complements are unique in distributive lattices, not necessarily so in more general lattices. When all complements are unique, we write complementation as a unary relation (operation) '; this relation is not necessarily total even in distributive lattices with 0,1 . A Boolean lattice, or Boolean algebra, is a distributive lattice with 0,1 , in which every element has a complement. Any subset lattice $L=2^{X}$ is a Boolean algebra, with $0=\emptyset$ and $1=X$.

Definition $2 A$ (locally finite) phenomenological theory $\mathcal{P}$ is a set $\mathcal{M}$ of disjoint finite sets $M$, together with a set $\Omega$ of functions ("states") $\omega$ from (all of) $\cup_{M \in \mathcal{P}} M$ to $[0,1]$ such that for any $M, \sum_{x \in M} \omega(x)=1$.

$M$ are the possible measurements; taking them to be disjoint means we are not allowing any a priori identification of outcomes of different measurement procedures. $\Omega$ is the set of phenomenologically admissible compendia of probabilities for measurement outcomes. 
The set $\mathcal{M}$ is an example of what Foulis calls a "test space": a set $\mathcal{T}$ of sets $T$, where $T$ may be interpreted as operations, (tests, procedures, whatever you want to call them) and the elements $t \in T$ as outcomes of these operations. (Without the interpretation, these are better known in mathematics as hypergraphs or set systems.) Call the set of all outcomes $\Lambda:=\cup T$. In general test spaces the $T$ need not be disjoint; here they are. Foulis calls such test spaces "semiclassical." (Sometimes a weak requirement of irredundancy, that none of these sets is a proper subset of another, is imposed on test spaces; it is automatic here.) States on test spaces are functions $\omega: \Lambda \rightarrow[0,1]$ such that $\sum_{t \in T} \omega(t)=1$ for any $T$. It is only when a phenomenological theory is defined as a set of states on a general $\mathcal{T}$, where a given outcome may occur in different measurements, that the question of contextuality (does the probability of an outcome depend on the measurement it occurs in?) arises at the phenomenological level. By not admitting such a primitive notion of "same outcome," but distinguishing outcomes according to the measurements they occur in, the construction we make will guarantee noncontextuality of probabilities even at the later stage where the theory is represented by a more abstract structure in which the elements (effects, or operations) that play the role of outcomes may occur in different operations. Though the rest of our discussion ignores it, the question of whether there can be convincing reasons for admitting a primitive notion of "same outcome" (based perhaps on some existing theory in terms of which the operations and experiments of our "phenomenological theory" are described) is worth further thought. A related point is that test spaces provide a framework in which we can implement a primitive notion of two outcomes of different measurements being the same, but we cannot implement a notion of two outcomes of the same measurement being the same (up to, say, arbitrary labeling). A formalism in which one can is that of E-test spaces (the E is for effect). These are sets, not of sets of outcomes, but of multisets of outcomes. Multisets are just sets with multiplicity: each element of the universe is not just in or out of the set, but in the set with a certain nonnegative integer multiplicity. Where sets can be described by functions from the universe to $\{0,1\}$ (their characteristic functions), multisets are described by functions from $U$ to $\mathbb{N}$. The set of resolutions of unity in an effect algebra, shorn of its algebraic structure, is an E-test space (whence the name). Not all E-test spaces are such that an effect algebra can be defined on them; those that are are called algebraic. Sufficiently nice E-test spaces are prealgebraic, and can be completed to be algebraic by adding more multisets without enlarging the universe (underlying set of outcomes).

To each phenomenological theory we may associate a set of Boolean algebras, one for each measurement. We will call this set of Boolean algebras the "phenomenological logic" of the theory; note, though, that it is independent of the state-set $\Omega$. These are just the subset lattices of the sets $M$, or what I previously called the "propositional logics" of statements about the results of the measurements. We will distinguish them by subscripts on the connectives saying which measurement is referred to, e.g. $\wedge_{M}$ (although this is redundant due to the disjointness of the measurements).

The phenomenological states $\omega$ of $\mathcal{P}$ naturally induce states (which we will also call $\omega$ ) on the logic of $\mathcal{P}$, via $\omega(\{a\})=\omega(a), \omega(X)=\sum_{x \in X} \omega(x)$. They will satisfy $\omega(M)=1$ for each $M$, and $\omega(\emptyset)=0$. We have, for example ( $x$ and $y$ are now subsets of outcomes), 
$\omega\left(x \vee_{M} y\right)=\omega(x)+\omega(y)-\omega\left(x \wedge_{M} y\right)$, (which is equivalent to its dual). We call the elements of the Boolean algebras of a phenomenological logic events, and we will refer to the set of events of $\mathcal{P}$ as $\mathcal{V}$.

Definition 3 Events e, $f$ are probabilistically equivalent, $e \sim f$ in a phenomenological theory if they have the same probability under all states: $\forall \omega \in \Omega, \omega(e)=\omega(f)$.

$\sim$ is obviously an equivalence relation (symmetric, transitive, and reflexive). Hence we can divide it out of the set $\mathcal{V}$, obtaining a set $\mathcal{V} / \sim=: \mathcal{E}(\mathcal{P})$ of equivalence classes of events which we will call the effects of the theory $\mathcal{P}$. (We have dependence on $\mathcal{P}$, rather than just $\mathcal{M}$, because although $\mathcal{V}$ depends on $\mathcal{M}$ but not $\Omega, \sim$ depends also on $\Omega$. ) Call the canonical map that takes each element $a \in \mathcal{V}$ to its equivalence class, "e." The images $e(M)$ of the measurements $M$ under $e$ are "measurements of effects." Together they form an $E$-test space as defined above (a set of multisets). We now define on this space another "logic" which is, at least as far as possible, the simultaneous "image" under the map $e$ of each of the Boolean algebras $M$. To this end, we introduce a binary operation $\oplus$ on the effect space.

Definition $4 e_{1} \oplus e_{2}:=e\left(a \vee_{M} b\right)$ for some a such that $e_{1}=e(a), b$ such that $e_{2}=e(b)$, and $M$ such that $a, b \in M$ but $a \cap b=\emptyset$.

If no such $a, b, M$ exist, $\oplus$ is undefined on the effect space. (If they do exist, we will say they witness the existence of $e_{1} \oplus e_{2}$.) As part of the proof of Theorem 1 we will show from the definition of the map $e$ via probabilistic equivalence and the behavior of probabilities with respect to $\vee_{M}$, that this definition is independent of the choice of $a, b, M$.

Let $\omega^{e}$ denote the function from the effects to $[0,1]$ induced in the obvious way by a state $\omega$ on the Boolean algebra: effects being equivalence classes of things having the same value of $\omega$, we let $\omega^{e}$ take each equivalence class to $\omega$ 's value on anything in it.

Definition $5 A$ set of states $\Omega$ on a WEA $\mathcal{E}$ is separating if for $x, y \in \mathcal{E}, x \neq y \Rightarrow \exists \omega \in$ $\Omega(\omega(x) \neq \omega(y))$.

Theorem 1 The set $\mathcal{E}(\mathcal{P})$ of effects of a phenomenological theory $\mathcal{P}$ with state-set $\Omega$,

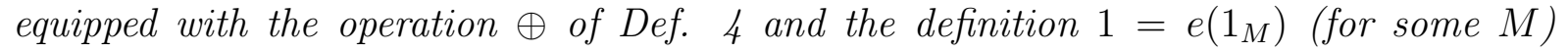
constitutes a weak effect algebra. There exist phenomenological theories for which this is properly weak, i.e. not an effect algebra. For all $\omega \in \Omega$ the functions $\omega^{e}$ defined above are states on the resulting weak effect algebra. $\Omega^{e}:=\left\{\omega^{e} \mid \omega \in \Omega\right\}$ is separating on $\mathcal{E}(\mathcal{P})$.

The proof is a straightforward verification of the axioms and the statements about states from the definition, and an example for the second sentence. 
Proof: We begin by demonstrating $\oplus$ is in fact a partial binary operation. This is done by verifying the independence, asserted above, of the definition of $\oplus$ from the choice of $a, b, M$ and of 1 from $M$. Suppose $e_{1}=e(a)=e(c), e_{2}=e(b)=e(d), a, b \in$ $M, c, d \in N, a \neq b, c \neq d, a \wedge_{M} b=0, c \wedge_{N} d=0$. Consider any state $\omega$ on the set of Boolean algebras which is also in $\Omega$, the states of our phenomenological theory. By the definition of $e, \omega(a)=\omega(c)$ and $\omega(b)=\omega(d)$; therefore $\omega(a)+\omega(b)=\omega(c)+\omega(d)$. Now $\omega\left(a \vee_{M} b\right)=\omega(a)+\omega(b)$ because $a \vee_{M} b=0$, and similarly $\omega\left(c \vee_{N} d\right)=\omega(c)+\omega(d)$. In other words, for any state $\omega \in \Omega, \omega\left(a \vee_{M} b\right)=\omega\left(c \vee_{N} d\right)$, so $a \vee_{M} b$ and $c \vee_{N} d$ are probabilistically equivalent, and correspond to the same effect.

Each Boolean algebra contains a distinguished element 1; by the definition of state on $\mathcal{P}$, these have probability zero, and one, respectively, in all states. Hence they each map to a single effect, and these effects we will call 0 and $u$ in the effect algebra (verifying later that $0=1^{\prime}$ in the weak effect algebra, so that it is consistent with the usual definition of 0 in a WEA). Of course, $\omega^{e}(1)=1$. It is also easy to see that $\omega^{e}(x \oplus y)=\omega^{e}(x) \oplus \omega^{e}(y)$. Hence the $\omega^{e}$ are states, as claimed. The set $\Omega^{e}$ is obviously separating. To be pedantic, suppose there exist effects $x, y$ having $\omega^{e}(x)=\omega^{e}(y)$ for all $\omega^{e} \in \Omega^{e}$. By the definition of $\omega^{e}, \omega^{e}(x)$ is the common value of $\omega$ on all $e$-preimages of $x$, and $\omega^{e}(y)$ is the common value of $\omega$ on all $e$-preimages of $y$. If these values are the same for all $\omega^{e}$, then the preimages of $x$ and of $y$ are all in the same equivalence class, so $x=y$. Hence, $\Omega^{e}$ is separating.

We now verify that $\oplus$ satisfies the weak effect algebra axioms.

(EA1) Strong commutativity: If $a, b \in M$ witness the existence of $x \oplus y$ as described in the definition of $\oplus$, by symmetry of $\vee_{M}$ and $\wedge_{M}$ (which enter symmetrically in the definition of $\oplus$ ) they also witness the existence of $y \oplus x$ and its equality with $x \oplus y$.

(WEA2) Weak associativity. Let $a, b \in M, e(a)=x, e(b)=y, a \cap b=\emptyset$, so that $a, b$ witness the existence of $x \oplus y$, and also let $c, d \in N$ and disjoint, $e(c)=z, e(d)=x \oplus y$, so $c, d$ witness the existence of $(x \oplus y) \oplus z$. Similarly let $b^{\prime}, c^{\prime} \in P$ witness the existence of $y \oplus z$ and $a^{\prime}, f \in Q$ witness the existence of $x \oplus(y \oplus z)$, so that $e\left(a^{\prime}\right)=x, e(f)=y \oplus z$, and $a^{\prime}, f$ are disjoint. Then $\omega^{e}(x \oplus y)=\omega(a) \oplus \omega(b)$ and $\left.\omega^{e}((x \oplus y)) \oplus z\right)=\omega(a)+\omega(b)+\omega(c)$. Also $\omega^{e}(y \oplus z)=\omega\left(b^{\prime}\right) \oplus \omega\left(c^{\prime}\right)=\omega(b) \oplus \omega(c)$, so $\omega^{e}\left((x \oplus(y \oplus z))=\omega\left(a^{\prime}\right) \oplus \omega(f)=\omega(a) \oplus\right.$ $\omega(b) \oplus \omega(c)$. But $\omega^{e}((x \oplus y) \oplus z)=\omega^{e}(x \oplus(y \oplus z))$ for all $\omega^{e}$ implies $(x \oplus y) \oplus z=x \oplus(y \oplus z)$ by the fact that $\Omega^{e}$ is separating.

(EA3) Define $e^{\prime}$ to be $e\left(a^{\prime}\right)$, for any $a$ such that $e(a)=e$, and $a^{\prime}$ is $a$ 's unique complement in the Boolean algebra of the measurement $M$ containing it. Since for any state, $\omega\left(a^{\prime}\right)=$ $1-\omega(a)$ and this probability is independent of $a$ as long as $e(a)=e, e^{\prime}$ as thus defined is independent of which $a$ is chosen. Moreover, since $a \wedge_{M} a^{\prime}=0 e \oplus e^{\prime} \equiv e(a) \oplus e\left(a^{\prime}\right)$ is defined and equal to $e\left(a \vee_{M} a^{\prime}\right)=e\left(1_{M}\right)=1$, so that ' as we just defined it satisfies (EA3).

(EA4) Note that $x \oplus 1$ is equal to $e\left(a \vee_{M} 1_{M}\right)$, for some $M$ containing $a$ and with unit $1_{M}$, where $a \wedge_{M} 1=0$ and $e(a)=x$. But each $M$ has a unique $a$ such that $a \wedge_{M} 1_{M}=0_{M}$, namely $0_{M}$. So an $x$ such that $x \oplus 1$ exists; it must be $e\left(0_{M}\right)=0$. 
This proves the first part of the theorem. We remark that $1^{\prime} \equiv e\left(1^{\prime}\right)=e\left(0_{M}\right)$, so defining 0 as $e\left(0_{M}\right)$ for any $M$ coincides with the usual effect algebra definition as $1^{\prime}$. We now construct the counterexample required by the second part.

Consider a phenomenological theory consisting of states on the two atomic Boolean algebras:

$$
\begin{aligned}
& M:\left(\begin{array}{ll}
a & b
\end{array}\right)\left(\begin{array}{lll}
f & \\
N: & \left(\begin{array}{ll} 
& c
\end{array}\right)
\end{array}\right)
\end{aligned}
$$

with the indicated $a, \ldots, g$ being atoms of the Boolean algebras involved ("elementary measurement outcomes"). The vertical lining-up of parentheses in (11) visually indicates conditions we will impose on the theory: that all states of our phenomenological theory respect $\omega\left(a \vee_{M} b\right)=\omega(c)$ and $\omega(f)=\omega\left(d \vee_{N} g\right)$; further, let our theory contain states with nonzero probability for each of $a, b, c, d, f, g$. There are plenty of perfectly good empirical theories satisfying these constraints, but $\oplus$ on the effect set of such a theory will not exhibit strong associativity: although $e(a) \oplus e(b)$ exists and is equal to $e(c)$, and $e(c) \oplus e(d)$ exists and is therefore equal to $(e(a) \oplus e(b)) \oplus e(d)$, no effect $h$ exists with $e(h)=e(b) \oplus e(d)$.

Conjecture 1 (Completion conjecture for WEA's) Let $\mathcal{E}$ be a WEA obtained from a phenomenological theory. A unique effect algebra $\overline{\mathcal{E}}$, which we call the completion of $\mathcal{E}$, can be constructed from $\mathcal{E}$ as follows. Whenever only one side of the associativity equation exists, impose the equation (extend $\oplus$ to contain the pair that would appear on the other side). This can also be characterized as the smallest effect algebra containing $\mathcal{E}$ as a sub-weak-effect-algebra (with the latter concept appropriately defined).

Thus the well-developed and attractive theory of effect algebras could be useful in this more general context. The adjunction of these new relations and the new resolutions of unity whose existence they imply is an interesting theoretical move. In constructing theories, we often suppose the existence of things that do not, at least initially, correspond to things in the available phenomenology. The idea of including all Hermitian operators as observables in quantum mechanics is an example; there has been much discussion of whether they are all operationally observable. This has motivated the search, often successful, for methods of measuring observables that had previously not been measured, and the development of a general theory of algorithmic procedures for measurement. The conjecture above might motivate the search for empirical methods of making measurements which would correspond to the additional resolutions of unity needed to make the initial WEA into an effect algebra. In any case, it is worth studying the nature of information processing and information theory (if the latter still makes sense) in properly weak effect algebras versus their completions.

We are now ready for a few remarks on the significance of Gleason's theorem (Gleason, 1957) in this context. Gleason's theorem says that in Hilbert space dimension greater than 
two, if mutually exclusive quantum measurement results are associated with mutually orthogonal subspaces of a Hilbert space, and exhaustive sets of such measurements to direct sum decompositions of the space into such subspaces, and if the probability of getting the result associated to a given subspace in a given measurement is independent of the measurement in which it occurs ("noncontextual") then the probabilities must be given by the trace of the product of the projector onto the given subspace with a density operator. A similar theorem resolutions of unity into orthogonal projectors replaced by resolutions into arbitrary positive operators has been obtained by Busch (1999), and independently by Caves, Fuchs, Mannes, and Renes (Fuchs, 2001a, b). In the next section we will see how this theorem is a case of a general fact about convex effect algebras.

Sometimes Gleason-type theorems are used to justify the quantum probability law. Then one must justify the assumptions that probabilities are noncontextual, and that they are associated with orthogonal decompositions, or positive resolutions of unity, on a Hilbert space. Although Theorem 1 gives structures (WEAs) much more general than Hilbert space effect algebras (or their subalgebras consisting of projectors), it automatically results in noncontextual probability laws. But he construction of WEAs in Theorem 1 starts from probabilities, so it would be circular to use it to justify noncontextuality in an appeal to Gleason's theorem to establish quantum probabilities. Rather, Theorem 1 says that we can elegantly, conveniently represent any empirical theory by a set of noncontextual probability assignments on a certain WEA (and, if the completion conjecture is correct, embed this in an effect algebra). In the case of quantum theory, this general recipe provides both the Hilbert space structure and the trace rule for probabilities, as a representation of the compendium of "empirical" probabilities (perhaps somewhat idealized by the assumption that any resolution of unity can be measured) of quantum theory.

The generalization of Gleason-like theorems to weak effect algebras, effect algebras, and similar structures are theorems characterizing the full set of possible states on a given such structure, or class of such structures. In the particular case of a Hilbert space effect algebra, the import of the B/CFMR theorem, from our operational point of view, is that the quantum states constitute the full state space of the "empirically derived" effect algebra. This is especially interesting since in other respects, the category of effect algebras probably does not have enough structure to capture everything we would like it to about quantum mechanics: for example, the natural category-theoretic notion of tensor product of effect algebras (Dvurečenskii (1995); see also Wilce (1994, 1998)), applied to effect algebras of finite dimensional Hilbert spaces, does not give the effect algebra of the tensor product Hilbert space (or of any Hilbert space), as one sees from a result in Fuchs (2001a) (a similar result involving projectors only is in Foulis and Randall (1981)). Possibly relatedly, a natural category of morphisms for convex effect algebras, those induced by positive (order-preserving) linear maps on the underlying ordered linear space (see below), is larger in the quantum case than the "completely positive" maps usually considered reasonable for quantum dynamics. Nevertheless for a given Hilbert space effect algebra, its set of all possible states is precisely the set of quantum states.

The role of Gleason-like results depends to some extent on point of view. In the project of 
exploiting the analogies between quantum states and Bayesian probabilities, they can play a nice conceptual role. Probabilities are, roughly, "the right way" (nonarchimedeanity issues aside) to represent uncertainty, and to represent rational preferences over uncertain classical alternatives. In this "Bayesian" project, it would be very desirable to see quantum states as "the right way" to deal with uncertainty in a nonclassical situation: the Hilbert space structure perhaps sums up the "nonclassicality of the situation," and the probabilities can be seen as just the consequence of "rationality" in that situation. This suggests that the "structure of the nonclassical situation" mentioned above might be described in terms of measurement outcomes (sometimes called "propositions" or "properties") having probability zero or one; then Gleason's theorem or analogues for other "property" structures, might give the set of possible probability assignments for such a structure. This is related to the "Geneva" approach to empirical theories (rooted in the work of Jauch and Piron on "property lattices").

\subsection{Convex effect algebras}

It is natural to take the space of operations one may perform as convex. This represents the idea that given any operations $M_{1}$ and $M_{2}$, we can perform the operation $\left(\lambda_{1} M_{1}, \lambda_{2} M_{2}\right)$ (where $\lambda_{i} \geq 0, \lambda_{1}+\lambda_{2}=1$ ) in which we perform one of $M_{1}$ or $M_{2}$, conditional on the outcome of flipping a suitably weighted coin (or, in more Bayesian terms, arrange to believe that these will be performed conditional on mutually exclusive events, to which we assign probabilities $\lambda_{1}, \lambda_{2}$, that we believe to be independent of the results of measurements on the system under investigation). If we looked at the coin face and saw the index " $i$ " and obtained the outcome $a$ of $M_{i}$, this should correspond to an outcome $\lambda a$ of $\left(\lambda_{1} M_{1}, \lambda_{2} M_{2}\right)$, and any state should satisfy $\omega(\lambda a)=\lambda \omega(a)$.

Similar assumptions may be made at the level of effect algebra. For effect algebras constructed via probabilistic equivalence, they will be consequences of the convexity assumptions on the initial phenomenological theory; this will be worked out elsewhere. One could also pursue the consequences of imposing a generalized convexity based on a more refined notion of "vector probabilities", or other representations of uncertainty by nonarchimedean order structures. Such generalized probabilities and utilities can result from Savage-like representation theorems for preferences satisfying "rationality" axioms but not certain technical axioms that make possible real-valued representations (LaValle and Fishburn, 1992, 1996; Fishburn and LaValle, 1998). We will avoid such complications, but knowing about them may clarify the role of some technical conditions in results to be discussed below.

Definition $6 A$ convex effect algebra is an effect algebra $\langle E, u, \oplus\rangle$ with the additional assumptions that for every $a \in E$ and $\alpha \in[0,1] \subset \mathbb{R}$ there exists an element of $E$, call it $\alpha a$, such that (C1) $\alpha(\beta a)=(\alpha \beta) a$, (C2) If $\alpha+\beta \leq 1$ then $\alpha a \oplus \beta a$ exists and is equal to $(\alpha+\beta) a$, (C3) $\alpha(a \oplus b)=\alpha a \oplus \alpha b$ (again, the latter exists), (C4) $1 a=a$. The mapping $a \mapsto \alpha a$ from $[0,1] \times E$ to $E$ is called the convex structure of the convex effect algebra. 
Gudder and Pulmannová (1998) showed that "any convex effect algebra admits a representation as an initial interval of an ordered linear space," and in addition if the set of states on the algebra is separating, the interval is generating. To understand this result, we review the mathematical notion of a "regular" positive cone (which we will just call cone); it is basic in quantum information science, e.g because the quantum states, the separable states of a multipartite quantum system, the completely positive maps, the positive maps, unnormalized in each case, form such cones.

Definition 7 A positive cone is a subset $K$ of a real vector space $V$ closed under multiplication by positive scalars. It is called regular if it is (a) convex (equivalently, closed under addition: $K+K=K$ ), (b) generating $(K-K=V$, equivalently $K$ linearly generates $V$,) (c) pointed $(K \cap-K=\emptyset$, so that it contains no nonnull subspace of $V)$, and (d) topologically closed (in the Euclidean metric topology, for finite dimension).

Such a positive cone induces a partial order $\geq$ on $V$, defined by $x \geq_{K} y:=x-y \in K$. $\left(V, \geq_{K}\right)$, or sometimes $(V, K)$, is called an ordered linear space. The Hermitian operators on a finite-dimensional complex vector space, with the ordering induced by the cone of positive semidefinite operators, are an example. (A relation $R$ is defined to be a partial order if it is reflexive $(x R x)$, transitive $(x R y \& y R z \Rightarrow x R z)$ and antisymmetric $((x R y \& y R x) \Rightarrow x=y$.) The partial orders induced by cones have the property that they are "affine-compatible": inequalities can be added, and multiplied by positive scalars. If one removes the requirement that the cones be generating, cones are in one-to-one correspondence with affine-compatible partial orderings. In fact, the categories of real vector spaces with distinguished cones, and partially ordered linear spaces, are equivalent.

We pause to motivate some of the seemingly technical conditions of regularity. A regular cone may represent the set of unnormalized probability states of a system, or a set of specifications of expectation values of observables. The normalized states may be generated by intersecting it with an affine plane not containing the origin. Convexity is fairly clearly motivated by operational considerations, such as those in the definition of convex effect algebra above, or in the desire to have a normalized state set given by intersecting the cone with an affine hyperplane be convex. Topological closure is required so that the cone has extreme rays, and the convex sets we derive by, for instance, intersecting it with an affine hyperplane, will have extreme points if that intersection is compact; then the Krein-Milman theorem states that these extreme points convexly generate the set. (An affine hyperplane is just a translation of a subspace: for $d=3$, a 2 -d hyperplane is a plane in the sense of high school geometry.) In "empirically motivated" settings such as ours, in which the metric on the vector space will be related, via probabilities, to distinguishability of states or operations, limit points can be as indistinguishable as you want from things already in the cone, so closing a cone cannot have empirically observable effects, and may as well be done if it is mathematically convenient. In the presence of some of the other assumptions, pointedness ensures that the intersection with an affine plane can be compact. Its appearance in the representation theorem for convex effect algebras (presumably essentially because the convex sets one gets via states tend to be compact 
intersections of an affine "normalization" plane with such a cone) is one "operational" justification for pointedness. Pointedness also has a clear geometric interpretation: if the subspace $K \cap-K$ is one-dimensional, instead of a "point" at zero the cone could have an "edge," which is why nonpointed cones are often referred to as "wedges"; of course $\operatorname{dim}(K \cap-K)>1$ is also possible for a nonpointed cone. The property of being generating is often appropriate because any non-generating cone generates a subspace, and we may as well work there. When several cones are considered at once, this might no longer be appropriate.

An initial interval in such a space is an interval $[0, u]$ defined as the set of things between zero and $u$ in the partial ordering $\geq_{K}$, i.e. $\left\{x \in V: 0 \leq_{K} x \leq_{K} u\right\}$. It is generating if it linearly generates $V$. It can be viewed as a convex effect algebra by letting $\oplus$ be vector addition restricted to $[0, u]$ and the convex structure be the restriction of scalar multiplication. The representation theorem says any convex effect algebra is isomorphic (as a convex effect algebra) to some such linear convex effect algebra (via an affine map). In finite-dimensional quantum mechanics the vector space and cone are $H_{d}$ and the positive semidefinite cone, and the interval referred to in the representation theorem is $[0, I]$.

In addition to the requirements for states on an effect algebra, states on a convex effect algebra must satisfy $\omega(\lambda a)=\lambda \omega(a)$. The set of all possible states on a convex effect algebra may be characterized via a version of Lemma 3.3 of Gudder. Pulmannová. Bugaiski. and Beltrametti (1999), which describes it for linear effect algebras $[0, u]$. First, some definitions. The dual vector space $V^{*}$ for real $V$ is the space of linear functions ("functionals") from $V$ to $\mathbb{R}$; the dual cone $K^{*}$ (it is a cone in $V^{*}$ ) is the set of linear functionals which are nonnegative on $K$. Then $\Omega([0, u])$, the set of all states on $[0, u]$ when the latter is viewed as a convex effect algebra, is precisely the restriction to $[0, u]$ of the set of linear functionals $f$ positive on $K$ and with $f(u)=1$ ("normalized" linear functionals). The restriction map is a bijection. Viewing things geometrically, the states (restricted functionals) are in one-toone correspondence with the (unrestricted) functionals in the intersection of $K^{*}$ with the affine plane in $V^{*}$ given by $f(u)=1$. Since any linear functional on the $d^{2}$-dimensional vector space $H_{d}$ of Hermitian operators on $\mathbb{C}^{d}$ has the form $X \mapsto \operatorname{tr} A X$ for some $A$, while the dual to the positive semidefinite cone in $H_{d}$ is the set of such functionals for which $A \geq 0$ (i.e., the positive semidefinite cone is self-dual $\left.\left(K=K^{*}\right)\right)$ this Lemma tells us that the states of a finite-dimensional Hilbert space effect algebra are precisely those obtainable by tracing with density matrices $\rho$; in other words, the Gleason-type theorem for POVMs is a case of this general characterization of states on convex effect algebras. This illustrates the power and appropriateness of this approach (and probably other convex approaches, in which similar characterizations probably exist) to empirical theories, and to problems in quantum foundations. Gleason's theorem itself cannot be established in this way, because the effect algebra of projectors is not convex. However, there may be a natural notion of "convexification" of effect algebras according to which $[0, I]$ is the convexification of the effect algebra of projectors. Interesting questions are then, which effect algebras can be convexified, and for which of those (as for the effect algebra of quantum projectors) convexification does not shrink the state-space. Conversely, we might ask for ways of identifying special subalgebras of effect algebras, composed of effects having 
special properties like "sharpness", perhaps having additional structure such as that of an orthoalgebra, and investigate the relation between state-sets of effect algebras and these sub-algebras.

\subsection{Sequential operations}

The operational approach I am advocating suggests that one consider what general kinds of "resources" are available for performing operations. Provided both system and observer are sufficiently "small" portions of the universe, it may be reasonable to suppose that the observer may use yet other subsystems (distinct from both observer and system) as an "apparatus" or "ancilla" to aid in the performance of these operations, that the apparatus may be initially independent of the system and observer, and that the combination of apparatus and system may be viewed as a system of the same general kind as the original system, subject to the same sort of empirical operational theory, with a structure, and a state, subject to certain consistency conditions with that of the original system. (Convexity is a case of this, the ancilla functioning as "dice.") It may be that in some limits some of these assumptions break down, but it is still worth investigating their consequences for several reasons: so that we can recognize breakdowns more easily, so that we may even acquire a theoretical understanding of when and why to expect such breakdowns, and because we may gain a better understanding of why empirical theories valid in certain limits (say, small observer, small apparatus, small system) have the kind of structure they do.

Besides convex combination, other such elementary combinations and conditionings of operations should probably be allowed: essentially, the set of operations should be extended to allow including them as subroutines in a classical randomized computation. (Of course, this will not always be appropriate; for example, in investigating or constructing theories that are not even classically computationally universal.) Among other things, this might get us the $\oplus$ operation previously obtained as the image of $\mathrm{OR}(\mathrm{V})$ in Boolean propositional logics about each operation's outcomes, "for free," as we can use classical circuitry to construct procedures whose outcomes naturally correspond to propositional combinations of the outcomes of other procedures, and will have the same probabilities as those combinations. This leads us to the consider the possibility that the set of possible operations be closed under conditional composition. This means that given any operation $M$, and set of operations $M_{\alpha}, \alpha \in M$, there is an operation consisting of performing $M$, and, conditional on getting outcome $\alpha$ of $M$, then proceeding to perform $M_{\alpha}$. This assumption is natural, but nevertheless substantive: one could imagine physical theories that did not satisfy it. Some outcomes might destroy the system, or so alter it that we can no longer perform on it all the procedures we could before. Nevertheless, it is worth investigating the structure of theories satisying the assumption (the theory of quantum operations being one such case). The structures obtained when conditional composition is not universally possible might turn out to be understandable as partial versions of those we obtain when it is always possible, or in some other way be easier to understand once the case of total conditional composability is understood. An operation in this framework, then, can be viewed as a tree with a single root node on top, each node of which 
is labelled by an operation and the branches below it labelled by the outcomes of the operation, except that the leaves are unlabelled (or redundantly labelled by the labels of the branches above them). The interpretation is that the root node is the first operation performed, and the labels of the daughters of a node indicate the operation to be performed conditional on having just obtained the outcome which labels the branch leading to that daughter.

From now on, we mean by phenomenological theory a sequential phenomenological theory, i.e. one closed under conditional composition. If we extend a phenomenological theory via this requirement, the new outcome-set contains all finite strings of elements of the old outcome set. Given closure under conditional composition, a given string can now appear in more than one measurement. In order that the construction of dividing out operational probabilistic equivalence can work, we will have to require that the empirical probability of the string be noncontextual. We will also use a different notion of probabilistic equivalence: $x \sim y$ iff for any $a, b, \omega(a x b)=\omega(a y b)$, where $x, y, a, b$ are outcome-strings. In our context the noncontextuality assumption can actually be derived from the disjointness of "elementary" operations (those not constructed via composition) and the assumption that the choice of operation at node $n$ of the tree describing an operation constructed via conditional composition cannot affect the probabilities of outcomes corresponding to paths through the tree not containing node $n$. This is how one might formalize a generalization of the "no Everett phone" requirement suggested in Polchinski's Polchinski (1991) article on Weinberg's nonlinear quantum mechanics: the probability of an outcome sequence cannot depend on what operation we would have done had some outcome in this sequence not occurred.

With suitable additional formalization of the notion of phenomenological operational theory, and appropriate definitions of $\oplus$ and a sequential product on the resulting equivalence classes, one can prove that dividing probabilistic equivalence out of such a set of empirical operations, in a manner similar to the construction of weak effect algebras via probabilistic equivalence, gives what I will call a weak operation algebra. The details will be presented elsewhere. Here I will exhibit the quantum-mechanics of operations as a case of a general structure, an operation algebra (OA), which I view as the analogue, for operations, of an effect algebra. The structure will be related to the notion of sequential effect algebra (SEA) studied by Gudder and Greechie (2000), but differ from it in important respects. It would be interesting to study when the set of effects of an OA forms a SEA.

Since this structure will be a partial abelian semigroup, with extra structure involving only the PAS operation $\oplus$, with a product meant to represent composition of operations, and additional axioms about how the two interact, we will discuss some more aspects of PASes (following Wilce (1998)) before defining operation algebras. The reader might want to keep in mind the algebra of trace-nonincreasing completely positive maps (with $\oplus$ as addition of maps and the product as composition of maps) as an example.

Recall that a PAS is a set with a strongly commutative and strongly associative partial binary operation $\oplus$ defined on it. Define a zero of a PAS as an element 0 such that for 
any $a, a \oplus 0=a$. (Uniqueness follows.) If a PAS does not have a zero, it is trivial to adjoin one; we henceforth include its existence as part of a PAS. A PAS is cancellative if $x \oplus y=x \oplus z \Rightarrow y=z$, positive if $a \oplus b=0 \Rightarrow a, b=0$. The relation $\leq$ on a PAS is defined by $x \leq y \Leftrightarrow \exists z \quad x \oplus z=y$. Part of Lemma 1.2 of Wilce (1998) is that in a cancellative, positive PAS $\leq$ is a partial ordering. In such a PAS, we define $T$ as the set of top elements of the partial ordering (i.e. $T=\{t \in \mathcal{O} \mid a \oplus t$ exists $\Rightarrow a=0\}$ ). In a cancellative PAS we define $x \ominus y$ as that unique (by cancellativity) $z$, if it exists, such that $y \oplus z=x$. Define a chain in a partially ordered set $P$ as a set $C \subseteq P$ such that $\leq$ restricted to $C$ is total.

Definition 8 An operation algebra $\mathcal{O}$ is a cancellative, positive PAS equipped with a total binary operation, the sequential product, which we write multiplicatively. With respect to the product, the structure is (OA5) a monoid (the product is associative) with (OA6) a unit 1 (semigroup is sometimes used as a synonym for this unital monoid structure). The remaining axioms involve the interaction of this monoid structure with the PAS structure. (OA7) $0 c=c 0=0$.

(OA8) $(a \oplus b) c=a b \oplus b c, a(b \oplus c)=a b \oplus a c$ (distributive laws).

$(O A 9) 1 \in T$.

(OA10) Every chain in $\mathcal{O}$ has a sup in $\mathcal{O}$.

Note that the sup mentioned in (OA10) is not necessarily in the chain. (OA10) says that $\mathcal{O}$ is chain-complete; this is (nontrivally, and I am not certain whether choice or other strong axioms are required in the infinite case) equivalent to saying it is complete, meaning that every directed subset of $\mathcal{O}$ has a sup in $\mathcal{O}$. (A poset $P$ is directed if for every subset $S$ of it, $P$ contains an element $x$ greater than or equal to everything in $S$.) The thinking behind (OA10) is that we are to conceive of the elements or "operations" in $\mathcal{O}$ as possible outcomes of procedures performed on a system, and each such outcome must be part of at least one exhaustive set of such outcomes. Given how the ordering is defined, it might seem natural therefore to require that all upward chains terminate; however, when there are sufficiently many operations (and also, but not only, if continuous sets of outcomes for a given operation are envisaged), as in the quantum case, it could be reasonable to allow (what is certainly possible in the quantum case) chains that do not terminate, but have a limit point (the sup mentioned in (OA10)).

Our structure is not an effect algebra because we do not assume it is (as a PAS) unital (i.e., has at least one unit). A unit of a PAS is an element $u$ such that for any $a$, there is at least one $b$ such that $a \oplus b=u$. In a cancellative, positive, unital PAS (equivalently, effect algebra) there is a unique unit (the sole element of the top-set $T$ ). Axiom (OA10) might need strengthening in order to obtain some of the results one would like. Notably, we would like to have a representation theorem in which the operations belong to a cone in a vector space (and thus belong to an algebra in one of the usual mathematical senses, of a vector space with an appropriate product). Aside from belonging to a cone, the special nature of the convex set of operations in such a representation theorem would be expressed by an additional requirement, deriving from (OA10), which would specialize to 
the trace-nonincreasing requirement in the case of the quantum operation algebra (and generalize the initial interval requirement in the analogous representation theorem for effect algebras).

We shall now show that quantum mechanics provides an example of this structure. We refer to the set of linear operators on $\mathbb{C}^{d}$ as $B\left(\mathbb{C}^{d}\right)$.

Proposition 1 The set of trace-nonincreasing completely positive linear maps on $B\left(\mathbb{C}^{d}\right)$, with the identity map $\mathcal{I}$ as 1 , the map $M$ defined by $M(X)=0$ for every $X$ as 0 , ordinary addition of maps as linear operators, restricted to the trace-non-increasing interval, as $\oplus$, and composition of maps as the sequential product, forms an operation algebra. Its top-set $T$ is the set of trace-preserving maps.

Proof: The commutativity (OA2) and associativity (OA1) of $\oplus$ and the behavior of 0 (OA7), and the unital monoid structure (OA5 and 6) are immediate. Cancellativity holds for addition in any linear space, so since $\oplus$ is here a restriction of addition on a linear space of linear maps, it is cancellative (OA3). It is positive (OA4) because $A+B=0 \Rightarrow A, B=0$ for $A, B$ in a pointed cone (such as the cone of completely positive linear maps). (OA8) follows from the distributivity of multiplication of linear operators over addition of linear operators. The top-set $T$ is the set of trace-preserving operations, which follows from the easy observation that if you add any operation besides the zero operation to a trace-preserving operation, the result is not trace-nonincreasing. (OA9) follows since the identity operation is trace preserving. (OA10) involves an elementary topological argument which will be omitted here.

We note the interpretation of $\oplus$ and $\ominus$ in terms of the HK representation of a map $\mathcal{A}$ in terms of operators $A_{i}$ (operators such that the map acts as $X \mapsto \sum_{i} A_{i} X A_{i}^{\dagger}$ ). Modulo irrelevant details of indexing, the $\mathrm{HK}$ representation sequence $A_{i}$ is a multiset $[A]$ of operators $A$ such that $A^{\dagger} A \leq 1 . \mathcal{A} \ominus \mathcal{B}$ exists if there are HK representations $[A],[B]$ such that $[B]$ is a submultiset of $[A]$. (Equivalently, there are standard HK representation sequences $A_{i}$ and $B_{i}$ such that $B_{i}$ is an initial segment of $A_{i}$, i.e. $\mathcal{B}(X)=\sum_{i} A_{i} X A_{i}^{\dagger}$ where $i$ ranges over the first $k A_{i}$.) Thus it is obvious that $\mathcal{A} \ominus \mathcal{B}$ will not always exist.

We define a weak operation algebra to satisfy all the above axioms except that associativity is replaced with weak associativity (whose statement is the same as in the definition of weak effect algebra). With suitable additional formalization of the notion of sequential phenomenological theory and sequential probabilistic equivalence, and definitions of $\oplus$ and sequential product on the equivalence classes, one can show:

Theorem 2 The set of equivalence classes obtained by dividing the notion of operational probabilistic equivalence defined above out of a phenomenological operational theory, has a natural weak operation algebra structure.

Note that if we have operational limits on conditional composition, as discussed above, we might accomodate that by modifying the notion of operation algebra (or WOA) to make 
the multiplicative monoid structure partial. It would then be interesting to investigate the conditions under which this partial structure is extendible to a total one (as well as the conditions under which a WOA can be completed to an OA).

We can add a convex structure to an OA with little difficulty. We just introduce a map of multiplication by scalars in $[0,1]$ (i.e. a map from $[0,1] \times \mathcal{O} \rightarrow \mathcal{O}$ ) such that the axioms $(\mathrm{C} 1-\mathrm{C} 4)$ of convex effect algebras hold, and also $(\alpha a) b=\alpha(a b)=a(\alpha b)$ (COA15). We expect such a structure to again emerge from an operational equivalence argument applied to a suitable notion of convex operational phenomenological theory.

\section{Dynamics and the combination of subsystems in operational theories}

The operation algebra approach sketched above implicitly includes a kind of dynamics, although without explicit introduction of a real parameter for time. Probably some operation algebras are extendible to have a notion of time. However, in the quantum operation algebra given above the assumption is that any completely positive evolution can be achieved. The time taken is neglected, and the temporal element of the interpretation is only the primitive one that when one measurement is done conditional on the result of another, it is thought of as done after the result of the first is obtained. A more substantial notion of time might be introduced in many different ways by adding structure to the operation algebra, e.g. by some consistent specification of how long each evolution takes, or by the assumption that each evolution can be done in any desired finite amount of time. The latter is a very strong assumption. In some cases, one might have a continuous semigroup structure related (with scheduling constraints) to their sequential product. A realistic consideration of these matters would involve a much more detailed account of the interactions between apparatus and system that are actually available. This is an important part of the project I propose, but I will not pursue it much here. It reminds us, though, of one of the important lessons of QIP for foundations mentioned in Section 2 that which operations are possible may depend on the resources available, and that the beautiful structures one sometimes encounters as operational theories may be idealized. In particular, much of the attempt to implement QIP involves struggling with the limitations imposed by the limited nature of the subsystems, and interactions, physics makes available. It is important to incorporate such limitations in operational structures. Barnum et al. (2002) is one approach to this, with the resources available for control and observation limited (for example) to those definable via a Lie subalgebra of the full Lie algebra $\mathfrak{s l}(d)$ appropriate to arbitary quantum operations. Physics includes much more than just Hilbert space: preferred bases or tensor product structures, symmetries, the whole business of representation theory. Another approach to involving this "more" in operational theories has been the inauguration, particularly in works such as Foulis (2000) and Wilce (2000), of a theory of group actions on empirical quantum logics.

An important part of the project of combining operational empirical logic and QIP ideas to investigate whether or not physics can provide an overarching structure unifying perspectives is to understand the operations available in an operational theory in terms of 
interactions with apparatus and/or environment. In particular, if we have a way, such as the tensor product in quantum mechanics, of describing the combination of apparatus $A$ and system $S$ as subsystems of a larger system $L$, we will probably want to require that the evolution induced on $S$ by doing an operation on the larger system is, under appropriate circumstances, one of the operations our theory describes as performable on the smaller system. "Appropriate circumstances" probably means that the apparatus should be initially independent of the system, which in turn requires that the notion of combination of subsystems have a way of implementing that requirement. Such assumptions bear close scrutiny, though, as they may be just the sort of thing that becomes impossible in certain limits. Some, such as (Ford et al., 2001), have argued for the physical relevance of some situations in which open systems are analyzed without the initial independence assumption. Independence works well in the case of completely positive quantum operations, though: indeed, all such operations can be implemented via a reversible interaction with apparatus. Consideration of categories, such as convex operation algebras and generalizations of these, that describe dynamics is probably the most promising way to investigate such questions. Possibly the category-theoretic notion of tensor product will be defined for these categories. One could then examine, for example, whether the tensor product of two Hilbert-space CP-operation algebras is the operation algebra of CP-maps on the tensor product of the Hilbert spaces. I doubt that it is.

To define the category-theoretic tensor product requires the notion of bimorphism. For categories whose objects are sets with additional structure, and whose morphisms are structure-preserving mappings we can define a bimorphism of $A, B$ as function $\phi: A \times B \rightarrow$ $T$, where $T$ is another object in the category, and $\phi$ has the property that for every $a \in A, \phi_{a}: B \rightarrow T$ defined via $\phi_{a}(b)=\phi(a, b)$ is a morphism, and similarly with the roles of $A, B$ reversed. In the category of vector spaces, for example, it is just a bilinear map.

Definition 9 The tensor product $A \otimes B$ is a pair $(T, \tau)$, where $T$ is another object in the category (also often called the tensor product) and $\tau: A \times B \rightarrow T$ is a bimorphism, and any bimorphism from $A \times B$ factors through $T$ in a unique way, and $T$ is minimal among objects for which such a $\tau$ exists.

To say $\tau$ factors through $T$ in a unique way is just to say that for any bimorphism $\beta: A \otimes B \rightarrow V$, there is a unique $\phi: T \rightarrow V$ such that $\beta$ is $\tau$ followed by $\phi$. Minimality in a set means not a subobject of any object in the set. Probably the uniqueness of the factorization is therefore redundant.

There is an "operational" motivation of this construction when it is applied to categories like effect algebras, operation algebras, etc...: it implements the notion that the two structures being combined appear as potentially "independent" subsystems of the larger system, in a fairly strong sense that one can do any operation (or get any outcome) on one subsystem while still having available the full panoply of operations (outcomes) on the other.

The category-theoretic tensor product of ordered linear spaces (vector spaces with dis- 
tinguished regular cones) is not well defined: more structure is needed. More precisely, while various constructions having the universal property (all bimorphisms factor through them) can be made, there is not a unique minimal one.

For a variety of operational structures one might use to describe quantum mechanical statics, including test spaces, orthoalgebras, and effect algebras, the tensor product is not the corresponding operational structure for the tensor product of Hilbert spaces. This could indicate that the structure describing statics requires more specialized axioms, still consistent with quantum mechanics, and then the tensor product in this new category, call it $\mathcal{Z}$, will come out right in the Hilbert space case. It could also be that the difficulty is the static nature of the categories. Indeed, the category-theoretic tensor product of test spaces or effect algebras includes measurements whose performance would seem to involve dynamical aspects. These are measurements describable as the performance of a measurement $M$ on system $A$, followed by the performance of a measurement $M_{\alpha}$, on $B$, where which measurement $M_{\alpha}$ is performed is conditional on the outcome $\alpha$ of the $A$-measurement. The the tensor product of effect algebras must contain all product outcomes, and it can be characterized as the effect algebra "generated" by requiring that it contain all the "1-LOCC" (local operations with one round, in either direction, of classical communication) measurements just described. Fuchs' (2001a) "Gleason-like theorem for product measurements" effectively does this construction for the case of Hilbert effect algebras. It is fairly elementary to show that the tensor product of EA's can also be characterized as the minimal "influence-free" effect algebra containing all product measurements (i.e. in which we can do all pairs of measurements one on $A$, one on $B$, with no communication). Freedom from influence of $B$ on $A$ means that for all states on the object, the probabilities of the outcomes of an $A$ measurement, performed together with an independent $B$ measurement, cannot be affected by the choice of measurement on $B$. Influence freedom means freedom from influence in both directions. Both of these characterizations provide strong operational motivation for the category-theoretic tensor product in this situation. Each is easily established starting from the other, and a similar construction of a "directed" product, in which 1-LOCC operations are allowed in one direction only, rules out "influence" in the direction opposite the communication. These things are also true, and were in fact first established for, test spaces (Foulis and Randall, 1981) and orthoalgebras Bennett and Foulis (1993).

The difficulty, in the quantum case, is that the tensor product of orthoalgebras or effect algebras, while it must contain measurements of effects that are tensor products of Alice and Bob effects, and, through addition of effects, all separable effects, does not contain "entangled" Alice-Bob effects. The separable effects span the same vector space $B\left(\mathbb{C}^{d} \otimes \mathbb{C}^{d}\right) \cong H_{d^{2}}$ of $d^{2} \times d^{2}$ Hermitian matrices (where A,B both have dimension $d$ ) as the full set of effects on $\mathbb{C}^{d} \otimes \mathbb{C}^{d}$, but they are the interval $[0, I]$ in the separable cone, not the interval $[0, I]$ in the positive semidefinite cone. Consequently the available states, while they must be linear functionals of the form $A \mapsto \operatorname{tr} A X$ for $d^{2} \times d^{2}$ Hermitian $X$, are the normalized members of the separable cone's dual, rather than of the positive semidefinite cone's dual, so $X$ in the functional $A \mapsto \operatorname{tr} A X$ is not necessarily positive semidefinite. The separable cone being properly contained in the positive semidefinite one, its dual 
properly contains the positive semidefinite one's dual, so that not only are we restricted to fewer possible measurements, but their statistics - even those of independent $A, B$ measurements - can be different from the quantum ones (although all quantum states are also possible states). Stated in more quantum information-theoretic terms: some nonpositive operators $X$ are nonpositive in ways that only show up as negative probabilities or nonadditivity when we consider entangled measurements: since in the effect-algebra or orthoalgebra tensor product we don't have entangled measurements available to "directly detect" this nonpositivity, these are admissible states on these tensor products. Indeed, as observed in Wilce (1992), they are isomorphic to the Choi matrices (block matrices whose blocks $M_{i, j}$ are $\left.T(|i\rangle\langle j|)\right)$ of positive, but not necessarily completely positive, maps $T$ (although the normalization condition (trace-preservation) appropriate for such maps is different from the (unit trace) normalization condition appropriate for states). Of course, the nonpositivity of the operator can be "indirectly detected" by tomography using separable effects, since these effects span the space of Hermitian operators.

One obvious solution to the problem would be to introduce axioms that would prohibit this divergence between the existence of entangled states and nonexistence of entangled measurements. Mathematically, this divergence reflects the important fact that the positive semidefinite versus separable effect algebras on $\mathbb{C}^{d} \otimes \mathbb{C}^{d}$ are differentiated by the properties of the corresponding cones: the former, but not the latter, being self-dual. Self-duality is a natural and powerful mathematical requirement on cones, but a very strong, and arguably not operationally motivated, one. Self-duality is an important part of the essence of quantum mechanics, so we should strive hard to understand its operational motivation and implications. The cones for classical effect algebras can also be self-dual: e.g. the algebra of fuzzy sets of $d$ objects. An axiom related to self-duality, violated by the tensor product of Hilbert effect algebras, is the "purity is testability axiom." We develop some concepts before formulating it.

Definition 10 An effect-algebra theory is a pair $\langle\mathcal{E}, \Upsilon\rangle$ where $\mathcal{E}$ is an effect algebra, $\Upsilon$ a convex set of states on that effect algebra. An effect $t$ passes a state $\omega$ if $\omega(t)=1$. An effect $t$ is a test for $\omega$ in theory $\langle\mathcal{E}, v\rangle$ if $t$ passes $\omega \in \Upsilon$ and for no state $\sigma \neq \omega, \sigma \in \Upsilon$, does $t$ pass $\sigma$. A state $\omega \in \Omega$ is testable in $\langle\mathcal{E}, \Omega\rangle$ if a test for it exists in $\mathcal{E}$.

Note that $\Upsilon$ may be smaller than $\Omega(\mathcal{E})$, the set of all possible states on $\mathcal{E}$. We now assume effect algebras are convex. If two tests pass $\omega$, so does any mixture of those tests. Let $t$ be a test for $\omega$, then for $\sigma \neq \omega,(\lambda \omega+(1-\lambda) \sigma)(t)=\lambda \omega(t)+(1-\lambda) \sigma(t)<1$, i.e. $t$ cannot test any mixture of $\omega$ with something else. Although a test thus tests a unique state, it is not necessarily the case that a testable state has a unique test. Let $t$ test $\omega$; suppose $\omega=\lambda \sigma+(1-\lambda) \tau$. Then $1=\omega(t)=\lambda \sigma(t)+(1-\lambda) \tau(t)$. This implies that $\sigma(t)=\tau(t)=1$, hence by the fact that $t$ tests $\omega, \sigma=\tau=\omega$. In other words, only pure (extremal) states can be testable. We will be interested in Axiom 1: all pure states are testable. To study the consequences of this axiom, we introduce a basic notion in convex sets.

Definition 11 A face of a convex set $C$ is an $F \subseteq C$ such that for every point $p \in F$, 
all points in terms of which $p$ can be written as a convex combination are also in $F$. In other words, for $\lambda_{i} \geq 0, \sum_{i} \lambda_{i}=1, \sum_{i} \lambda_{i} x_{i} \in F \Rightarrow\left(\forall i, x_{i} \in F\right)$.

Thus a face of $C$ is the intersection of the affine plane it generates with $C$. The set of faces, ordered by set inclusion, forms a lattice. This lattice characterizes the convex set. (up to affine isomorphism, which is the proper notion of isomorphism for convex sets since affine transformations $y \mapsto A y+b$ commute with convex combination).

Proposition 2 The theory $\left\langle\mathcal{E}\left(\mathbb{C}^{d}\right) \otimes \mathcal{E}\left(\mathbb{C}^{d}\right), \Upsilon\right\rangle$ violates Axiom 1 unless $\Upsilon$ is contained in the set of separable states. In particular, $\left\langle\mathcal{E}\left(\mathbb{C}^{d}\right) \otimes \mathcal{E}\left(\mathbb{C}^{d}\right), \Omega\left(\mathcal{E}\left(\mathbb{C}^{d}\right) \otimes \mathcal{E}\left(\mathbb{C}^{d}\right)\right)\right\rangle$ violates it.

Proof: The proof proceeds by showing that the only states testable in $\mathcal{E}\left(\mathbb{C}^{d}\right) \otimes \mathcal{E}\left(\mathbb{C}^{d}\right)$ are pure product states. Then if Axiom 1 is satisfied, the extremal states of $\Upsilon$ are product states, so $\Upsilon$ is a face of the convex set of separable states. Let $\operatorname{tr} X=1$ and $\langle\chi|\langle\psi|X| \psi\rangle| \chi\rangle \geq 0$ for all product states $|\phi\rangle|\chi\rangle$, so that $A \mapsto \operatorname{tr} A X$ is a state. Testability means there is a separable $A$ with trace between zero and one (separable effect) such that: $1=\operatorname{tr} A X$. The first requirement on $A$ says that $A=\sum_{i} \lambda_{i}\left|\chi_{i}\right\rangle\left|\psi_{i}\right\rangle\left\langle\psi_{i}\right|\left\langle\chi_{i}\right|$ (for $\left.\lambda_{i}\right\rangle$ $0, \sum_{i} \lambda_{i} \leq 1,\left|\chi_{i}\right\rangle,\left|\psi_{i}\right\rangle$ normalized). Thus $\operatorname{tr} A X=1$ becomes $\sum_{i} \lambda_{i}\left\langle\chi_{i}\left|\left\langle\psi_{i}|X| \psi_{i}\right\rangle\right| \chi_{i}\right\rangle=1$, which can only hold if one of the $\lambda_{i}=1$, and for that $i,\left\langle\chi_{\mid}\left\langle\psi_{i}|X| \psi_{i}\right\rangle \mid \chi_{i}\right\rangle=1$. Then (dropping the subscript) $X=|\chi\rangle|\phi\rangle\langle\phi|\langle\chi|+X^{\pi, \perp}+X^{\perp, \pi}+X^{\perp, \perp}$. This is a resolution of $X$ into components in four subspaces of the space of operators on $\mathbb{C}^{d} \otimes C^{d}$ : the space $\pi, \pi$ of operators on the one-dimensional Hilbert space $\pi$ spanned by the pure product state, the space $\pi, \perp$ of operators taking $\pi$ to $\pi^{\perp}$, the space $\perp, \pi$ going the other way, and the space $\perp, \perp$ of operators on $\pi^{\perp}$. The middle two pieces are manifestly traceless, so the last one must be traceless for $\operatorname{tr} X=1$ to hold. However, $\operatorname{tr} X^{\perp, \perp}=\sum_{i j}\langle i|\langle j|X| j\rangle| i\rangle$ in a product basis $|i\rangle|j\rangle$ for $\perp$. Each $\langle i|\langle j|X| j\rangle| i\rangle$ must be positive since $\operatorname{tr} X^{\perp, \perp} A=\operatorname{tr} X A$ for $A \in \perp, \perp$. So for $X^{\perp, \perp}$ to be traceless, they must all be zero, and $X=|\chi\rangle|\phi\rangle\langle\phi|\langle\chi|$ plus possibly some traceless stuff which does not affect the induced state.

Note that we can have a theory on $\mathcal{E}\left(\mathbb{C}^{d}\right) \otimes \mathcal{E}\left(\mathbb{C}^{d}\right)$ satisfying the axiom of testability, but only if the state space is contained in the dual of the cone generated by the effect algebra. This suggests that the axiom, if required of the full state space $\Omega$ of an effect algebra, is pushing us towards the idea that the cone be self-dual.

Testability is very natural, and has a long history in quantum logic (e.g. Mielnik (1969) and probably Ludwig (1983a; 1985)). Theories which are the full state spaces of linear effect algebras that are initial intervals in self-dual cones satisfy it. This axiom makes contact with the "property lattice" quantum logics of Jauch (1968) and Piron (1976). (See Valckenborgh (2000, pp. 220-221)). It is also related to Rüttiman's Rüttiman (1981) notion of "detectable property." Jauch and Piron's notion of property roughly corresponds to effects (or the analogues in other quantum structures, since most of their work was done before effect algebras were formalized in the quantum logic community) $e$ which can have probability one in ("pass") some states. Those states are said to "possess the property $[e] "$. Properties are equivalence classes of effects that pass the same set of 
states. They construct a lattice of properties for an empirical theory (set of states on some quantum structure).

Axiom 1 relates the lattice of faces of a convex set of states on an effect algebra to the property lattice of that theory. The extremal states are minimal in the face lattice; the axiom says there are "minimal properties" possessed by those states: minimal in the sense that no other state posesses them. I am not certain if this is minimality in the sense of Piron's property lattice, but it seems likely (perhaps under mild conditions). A generalization of Axiom 1 asserts, for each face of the state-set, the existence of a "property" of being in that that face (an effect passing the states of that face and no others). A similar axiom of Araki (1980) concerns "filters" for higher dimensional faces, but this also involves "projection postulate-like" dynamics associated with the filtering. Araki also uses, as an assumption, the symmetry or "reciprocity" rule, satisfied in the quantum-mechanical case, that can be formulated once a correspondence $\chi \leftrightarrow e_{\chi}$ between extreme states $\chi$ and tests $e_{\chi}$ for them has been set up. Reciprocity requires that $\chi\left(e_{\phi}\right)=$ $\phi\left(e_{\chi}\right)$. It is not clear to me whether the extreme states $\rightarrow$ effects correspondence must be one-to-one instead of many-to-one in order to be able to formulate the axiom, or whether one-to-oneness might be a consequence of it. (Faces play an important role in Ludwig's work as well, as do statements reminiscent of Axiom 1, so Ludwig's argument may turn out to be similar.)

Araki credits Haag for emphasizing to him the importance of the reciprocity axiom. In the second edition of his book, Haag (1996) includes a informal discussion of the foundations of quantum mechanics based on the convex cones framework. He, too, uses Axiom 1, and a generalization associating faces of the state space (one-to-one!) with "propositions." These "propositions" are effects passing precisely the states of the face, and minimal among such effects in the sense of a probabilistic ordering of effects $e_{1} \leq e_{2}:=\forall \omega \in$ $\Upsilon \omega\left(e_{1}\right) \leq \omega\left(e_{2}\right)$. This is a different strategy from the Jauch-Piron equivalence class one for getting uniqueness of the effect associated to a face, but it is closely related to it. Jauch and Piron were trying to get by with less reference to probabilities. Haag also uses the reciprocity axiom, which he argues imposes self-duality. ${ }^{2}$

Haag also gives some operational motivation for an additional assumption, that of homogeneity of the cone. This says that the automorphism group of the cone acts transitively on its interior. (For any pair $x, y$ of interior points, there is an automorphism taking $x$ to $y$.) Interpret cone automorphisms as conditional dynamics; then homogeneity, at least for self-dual cones, means that any state is reachable from any other by dynamics conditional on some measurement outcome. This is not self-evident but seems natural. If you can't prepare any state starting from any other state, with a nonzero probability of success, the state space might "fall apart" into pieces not reachable one from the other (orbits of the automorphism group). Or while some pieces might still be reachable from all others,

\footnotetext{
${ }^{2}$ Haag uses uses the notion of self-polarity, but for our type of cone, this is the same as self-duality. The polar of a convex body $C$ is the set of linear functionals $L$ such that $L(x) \leq 1$ for all $x \in C$; the polar of a cone is the negative of the dual cone, since whenever $L(x)$ is positive, $L\left(x^{\prime}\right)$ is greater than 1 for $x^{\prime}$ a large enough positive multiple of $x$. Since the negative of a cone is isomorphic to that cone, a self-polar cone is self-dual.
} 
going the other way might not be possible: there would be intrinsically irreversible dynamics, even conditionally. A more detailed study of operational theories whose effects are naturally represented in a non-homogeneous cone, or whose state-space generates one, would be desirable (either with or without self-duality). The "falling apart" into orbits of the automorphism group may be acceptable in a theory of a perspective involving radical limitations on our ability to prepare states: going from one orbit to another might require a more powerful agent than the one whose perspective is being considered, but the consequences of such an agent's actions might be observable by the less powerful agent. Entanglement is such a situation: the perspective of the set of local agents, even with the power to communicate classically, allows for pairs of states with different statistics for observables implementable by local actions and classical communication (LOCC), such that it is impossible, even conditional on a measurement outcome, to prepare one starting from the other via LOCC W. Dür (2000). The LOCC perspective of the local agents is not usually taken as a "subsystem" in quantum mechanics, so these sorts of perspectives can there be taken as derivative rather than fundamental; but perhaps in other situations nonhomogeneous perspectives could be more fundamental.

In finite dimensions, as Haag points out, homogeneous self-polar cones are known (e.g. (Vinberg, 1965)) to be isomorphic to direct products of the cones whose faces are the subspaces of complex, quaternionic, or real Hilbert spaces. (Extensions of these results to infinite dimensions are obtained in Connes (1974).) The factors in the direct product can be thought of as "superselection sectors;" classical theory would be recovered when the superselection sectors are all one-dimensional (at least in the complex and real cases). Araki (1980) obtains a similar theorem except the effects get represented as elements of a finite dimensional Jordan algebra factor. These are isomorphic to to $n \times n$ Hermitian matrices over $\mathbb{R}, \mathbb{C}$, or the quaternions $\mathbb{H}$, or a couple of exceptional cases (spin factors and $3 \times 3$ Hermitian matrices over the Cayley numbers). He also gives arguments for picking the complex case, based on the properties of composition of subsystems in the various cases. Araki's argument is that "independence" of the subsystems should be expressed by $\operatorname{dim} V=\left(\operatorname{dim} V_{1}\right)\left(\operatorname{dim} V_{2}\right)$ for the algebras. But, "essentially because the tensor product of two skew-Hermitian operators is Hermitian", we have $\operatorname{dim} V>$ $\left(\operatorname{dim} V_{1}\right)\left(\operatorname{dim} V_{2}\right)$ except in trivial cases, when we take the $V$ 's to be the algebras of Hermitian matrices over real Hilbert spaces $H_{1}, H_{2}$, and their tensor product. (A related requirement plays a similar role in Hardv $(2001 \mathrm{a}, \mathrm{b})$ ).) For $\mathbb{Q}$ there is not even a quaternionlinear tensor product. The bottom line is that "the complex field has the most pleasant feature that the linear span of the state space of the combined system is a tensor product of [the state spaces of the] individual ones." There are probably important operational and information-theoretic distinctions between the cases which merit closer study. In the real case, the key point is that in contrast to the complex case, states on the "natural" real composite system are not determined by the expectation values of local observables.

Like homogeneity, self-duality and reciprocity may be related to the coordination of perspectives into an overall structure. In a "spin-network" type of theory, the edges of a graph are labelled with representations of a Lie or quantum group $(\mathfrak{s u}(2)$, for spin networks), which are Hilbert spaces. The vertices are associated to "intertwiners" between 
those representations. A state might be associated with, say, a partition of the graph by a hypersurface cutting it into two parts, "observer" and "observed." If the hypersurface has two disconnected parts, the associated Hilbert space will be the tensor product of the ones associated with the parts; otherwise, the representation is made out of the representations labelling the cut edges, in a way determined by the intertwinings at the vertices between them. One has the same Hilbert space whichever piece one takes as "observer" vs. "observed." However, it is likely that the role-reversal between observer and observed corresponds to dualization, and the result that both correspond to the same Hilbert space will only hold in theories in which the structure describing a given perspective - here, the Hilbert space associated with the surface - is self-dual. To attempt to actually show something like this would involve a project of trying to construct "relational" theories like the Crane-Rovelli-Smolin theories, but with other empirical theories playing the role of Hilbert spaces and algebras of observables on them. A simple first example might be "topological classical field theories," if these can consistently be defined. In these general "pluralistic structures" coordinating perspectives, one might hope to find a role for selfduality and the reciprocity axiom, and perhaps homogeneity as well. For the different empirical structures associated with different surfaces to relate to each other in a "nice" way, it might be necessary that the structures be defined on self-dual cones or exhibit reciprocity. Another suggestion that bears more detailed investigation, perhaps also in the "relational" context since there time is sometimes taken as emergent, is due to Haag, who says, "[reciprocity] expresses a symmetry between "state preparing instruments" and "analyzing instruments" and is thus related to time-reversal invariance."

\section{Tasks and axioms: toward the marriage of quantum information science and operational quantum logic}

QIP emphasizes how the conceptual peculiarities of quantum mechanics allow us to perform tasks not classically possible. This suggests we these formulate tasks, or the associated concepts, in ways general enough to try to characterize different operational theories by whether or not these tasks can be performed in them, or by the presence or absence of conceptual phenomena such as: superposition, complementarity, entanglement, information-disturbance tradeoffs, restrictions on cloning or broadcasting, nonuniqueness

of the expression of states as convex combinations of extremal quantum states (versus the uniqueness classically), and so forth. An outstanding example involves cryptographic tasks (Fuchs, 2001a; Clifton et al., 2002). But even before the upsurge of interest in quantum information science, conceptual peculiarities like superposition (Bennett and Foulis, 1990) and nonunique extremal decomposition (Beltrametti and Bugaiski, 1993) were being generalized and studied in empirical/operational quantum logic.

Assumptions and tasks involving computation should also be investigated; In particular, it would be interesting to establish linkages between complementarity, or superposition, and computational speedup in a general setting. Or some conjunction of properties, such as no instantaneous communication between subsystems, common to quantum and classical mechanics, might be seen to imply no exponential speedup of brute-force search 
in a general setting. I claimed above that key aspects of using an operational point of view in foundational questions were understanding notions of subystems and system combination, and understanding dynamics. For information-processing or computation, both of these issues are of the utmost importance. Since the environment which induces noise in a system or the apparatus used by an information-processing agent must be considered together with the system, a notion of composite system is needed. And notions of composition of systems or of dynamics are basic to computational complexity, where the question may be how many bits or qubits are needed, as a function of the size of an instance of a problem (number of bits needed to write down an integer to be factored, say) to solve that instance. The very notion of Turing computability is based on a factorization of the computer's state space (as a Cartesian product of bits, or of some higher-arity systems), in terms of which a "locality" constraint can be imposed. The constraint is, roughly, that only a few of these subsystems can interact in one "time-step." The analogous quantum constraint allows only a few qubits to interact at a time. In general operational models, some notion of composition of systems, such as a tensor product, together with a theory describing what dynamics can be implemented on a subsystem, could allow for generalized circuit or Turing-machine models. Another way of obtaining a notion of resources is to specify a set of dynamical evolutions to which we ascribe unit cost, and a set of measurements viewed as computationally easy. More generally, we might specify a cost function on evolutions and measurements. A formal treatment will require us to say how we interface the given operational model with "classical" computation. We could specify a set of measurements-with-conditional-dynamics ("instruments") viewed as taking unit computational time, and allow the conditioning of further dynamics and measurement on the results of the measurement in question. Subtleties could arise in counting the computational cost of the classical manipulations required by such conditioning. Counting one elementary operation in some chosen classical computational model as costing the same as one in the general operational model is one reasonable approach (at least if the general model can simulate classical computation polynomially). More simply, perform the algorithm in the general operational setting by evolution without explicit measurement and classical control, and specify a "standard" measurement to be performed at the end (and a standard procedure for mapping the measurement result to the set of possible values of the function being computed). In non-query models, it is important that not just any measurement be allowed at the end, since if the dynamics consists of all effectalgebra endomorphisms, say, any computation can be done by making one measurement.

\section{Conclusion}

In this paper, I have promoted a particular project for harnessing the concepts of quantum information science to the task of illuminating quantum foundations. This project is to generalize tasks and concepts of information science beyond the classical and the quantum, to abstract and mathematically natural frameworks that have been developed for representing empirical theories; and to use these tasks and concepts to develop axioms for such theories, having intuitively graspable, perhaps even practical, meaning, or to develop 
a better understanding for the operational meaning of existing axioms. The main original technical contributions are Theorem 10 showing that any phenomenological theory naturally gives rise to a weak effect algebra, which is essentially the image of the propositional logic of statements about measurement outcomes under identification of probabilistically equivalent outcomes, and the introduction of the notions of operation algebra and weak operation algebra. These results and concepts are likely closely related to other work in operational quantum logic and the convex approach; I think they provide an appropriate framework for the project.

Within the scope of this project, I have emphasized what I think will be key aspects:

- A "perspectival, operational" approach to describing empirical theories, taking the probabilities of outcomes of operations an agent may do on the system as primary, and stressing that the structure of an empirical theory depends on the agent doing the operations as well as on the subystem the operations are done on.

- The structures of effect algebras and weak effect algebras, test spaces, and proposition lattices for observations, as well frameworks of "operation algebras" and "weak operation algebras" introduced here to encompass both dynamics and observables.

- A justification of weak effect and operation algebras through relations of "probabilistic equivalence," and "sequential probabilistic equivalence," as natural representations of very general classes of phenomenological theories. Gleason-type theorems take on a fresh aspect from this point of view.

- Convexity, and the resulting representations it makes possible in ordered linear spaces (real vector spaces with distinguished regular cones), and various mathematically natural axioms it suggests, such as homogeneity and self-duality.

- The significance of other natural operational desiderata, such as the idea that anything implementable via interaction with an independent ancilla should be considered an operation, or the idea that "evolve and then measure" should be considered a kind of measurement.

- The importance of attempts, like the Rovelli-Smolin "relational quantum mechanics," topological quantum field theories, spin networks, and "spacetime foams," to integrate agents' perspectives into a coherent whole, as special relativity does with its reference frames. The use of "integrability of perspectives into a coherent whole," as a possible source of axioms about the nature of perspectives (self-duality or homogeneity of the cones used to represent them?), how they combine (via tensor products or some other rule?), and so forth.

\section{Acknowledgments}

Discussions over the years with Carlton Caves, Dave Foulis, Chris Fuchs, Leonid Gurvits, Lucien Hardy, Richard Jozsa, Manny Knill, Eric Rains, Rüdiger Schack, Ben Schumacher, 
and Alex Wilce, among others, have influenced my thoughts on these matters.

\section{References}

Araki, H., 1980. On a characterization of the state space of quantum mechanics. Commun. Math. Phys. 75, 1-24.

Araki, H., Yanase, M., 1960. Measurement of quantum mechanical operators. Physical Review 120, 622-626, reprinted in Quantum Theory and Measurement, J.A. Wheeler and W.H. Zurek, eds., Princeton Univ. Press, 1983.

Banaszek, K., 2001. Fidelity balance in quantum operations. Phys. Rev. Lett. 86, 1366.

Barnum, H., 1990. The many-worlds interpretation of quantum mechanics: psychological versus physical bases for the multiplicity of "worlds", hardcopy available from the author on request.

Barnum, H., 1998. Quantum information theory UNM Doctoral Dissertation. Slightly corrected version available electronically at http://info.phys.unm.edu/papers/papers.html.

Barnum, H., 2001. Information-disturbance tradeoff in quantum measurement on the uniform ensemble (abstract). Proc. 2001 IEEE Intl. Symp. on Information Theory , 277. Electronic version of full paper available from the author.

Barnum, H., Caves, C. M., Fuchs, C. A., Jozsa, R., Schumacher, B. W., 1996. Noncommuting mixed states cannot be broadcast. Phys. Rev. Letters 76, 2818-2821.

Barnum, H., Hayden, P., Jozsa, R., Winter, A., 2001. On the reversible extraction of classical information from a quantum source. Proceedings of the Royal Society of London A 457, 2019-2039.

Barnum, H., Knill, E., Ortiz, G., Viola, L., 2002. Generalizations of entanglement based on coherent states and convex sets, arXiv.org e-print quant-ph/0207149.

Beltrametti, E. G., Bugajski, S., 1993. Decomposability of mixed states into pure states and related properties. Int J Theor Phys 32, 2235-2244.

Bennett, C. H., Brassard, G., Jozsa, R., Mermin, N. D., Peres, A., Schumacher, B. W., Wootters, W. K., 1994. Teleporting an unknown quantum state via dual classical and EPR channels. J. Mod. Opt. 41, 2307-2314.

Bennett, M. K., Foulis, D. J., 1990. Superposition in quantum and classical mechanics. Found. Phys. 20, 733-744.

Bennett, M. K., Foulis, D. J., 1993. Tensor products of orthoalgebras. Order 10, 271-282.

Bernstein, E., Vazirani, U., 1997. Quantum complexity theory. SIAM J. Comp. 26, 14741483. 
Bilodeau, D., 1996. Physics, machines, and the hard problem. J. Consciousness Studies $3,386-401$.

Bugajski, S., Gudder, S., Pulmannová, S., 2000. Convex effect algebras, state ordered effect algebras, and linear spaces. Rep. Math. Phys 45, 371-387.

Buhrman, H., Cleve, R., van Dam, W., 1997. Quantum entanglement and quantum communication Los Alamos ArXiV Preprint Archive quant-ph/9705033.

Busch, P., 1999. Resurrection of von Neumann's no-hidden-variables theorem, arXiv.org e-print quant-ph/9909073. New version in 2001.

Clifton, R., Bub, J., Halvorson, H., 2002. Characterizing quantum theory in terms of information-theoretic constraints. Studies in the History and Philosophy of Modern Physics this volume, also arXiv.org e-print quant-ph/0211089.

Clifton, R., Halvorson, H., 2001. Entanglement and open systems in algebraic quantum field theory. Studies in the History and Philosophy of Modern Physics 32, 1-31, also arXiv.org e-print quant-ph/0001107.

Connes, A., 1974. Caractérisation des espaces vectoriels ordonnés sous-jacents aux algèbres de von Neumann. Annales de l'Institut Fourier, Grenoble 24, 121.

Cooke, R. M., Hilgevoord, J., 1981. A new approach to equivalence in quantum logic. In: Beltrametti, E., van Fraassen, B. (Eds.), Current issues in quantum logic. Plenum, New York and London.

Deutsch, D., 1985. Quantum theory, the Church-Turing principle and the universal quantum computer. Proc R Soc London A 400, 97-117.

Deutsch, D., 1999. Quantum theory of probability and decisions. Proc R Soc London A 455, 93129-3137.

Deutsch, D., Jozsa, R., 1992. Rapid solution of problems by quantum computation. Proc R Soc London A 439 435, 553-558.

Dvurečenskij, A., 1995. Tensor product of difference posets and effect algebras. Int. J. Theor. Phys. 34, 1337-1348.

Feldman, D., Wilce, A., 1993. $\sigma$-additivity in manuals and orthoalgebras. Order 10, 383392.

Fishburn, P. C., LaValle, I. H., 1998. Subjective expected lexicographic utility with infinite state sets. J Mathematical Economics 30, 323-346.

Ford, G. W., Lewis, J. T., O’Connell, R. F., 2001. Quantum measurement and decoherence. Physical Review A 64, 032101.

Foulis, D. J., 1998. Mathematical metascience. J Natural Geometry 13, 1-50. 
Foulis, D. J., 2000. Representations on unigroups. In: Coecke, B., Moore, D., Wilce, A. (Eds.), Current research in operational quantum logic. Klüwer, Dordrecht.

Foulis, D. J., Bennett, M. K., 1994. Effect algebras and unsharp quantum logics. Found. Phys. 24, 1325-1346.

Foulis, D. J., Randall, C. H., 1981. Empirical logic and tensor products. In: Neumann, H. (Ed.), Interpretations and foundations of quantum mechanics: proceedings of a conference hold in Marburg 28-30 May 1979. Bibliographisches Institut, Zürich.

Fuchs, C., Peres, A., 1995. Quantum state disturbance vs. information gain: uncertainty relations for quantum information. Physical Review A 53, 2038.

Fuchs, C. A., 2001a. Quantum foundations in the light of quantum information, arXiv.org e-print quant-ph/0106166; to appear in Proceedings of the NATO Advanced Research Workshop on Decoherence and its Implications in Quantum Computation and Information Transfer, ed. A. Gonis.

Fuchs, C. A., 2001b. Quantum mechanics as quantum information (and only a little more), arXiv.org e-print quant-ph/0205039.

Fuchs, C. A., Peres, A., 2000. Quantum theory needs no "interpretation". Physics Today $53(3), 70==71$.

Gleason, A., 1957. Measures on the closed subspaces of a Hilbert space. Am. J. Math. Mech. 6, 885-894.

Greuling, R. G. H., 1989. Toward a formal language for unsharp properties. Found. Phys. 19, 931-945.

Gudder, S., Greechie, R., 2000. Sequential products on effect algebras, preprint.

Gudder, S., Pulmannová, S., 1998. Representation theorem for convex effect algebras. Commentationes Mathematicae Universitatis Carolinae 39, 645-659.

Gudder, S., Pulmannová, S., Bugajski, S., Beltrametti, E., 1999. Convex and linear effect algebras. Rep. Math. Phys 44, 359-379.

Haag, R., 1996. Local quantum physics. Springer, Berlin, revised 2nd edition. (First edn. is Springer, 1992.).

Hardy, L., 2001a. Quantum theory from five reasonable axioms, arXiv.org e-print quant-ph/0101012.

Hardy, L., 2001b. Why quantum theory?, arXiv.org e-print quant-ph/0111068. Contribution to NATO Advanced Research Workshop "Modality, Probability, and Bell's Theorem, Cracow, Poland 19-23.8.01.

Jauch, J. M., 1968. Foundations of quantum mechanics. Addison-Wesley, Reading, MA. 
Kôpka, F., Chovanec, F., 1994. D-posets. Mathematica Slovaca 44, 21-34.

LaValle, I. H., Fishburn, P. C., 1992. State-independent subjective expected lexicographic utility. J Risk and Uncertainty 5, 217-240.

LaValle, I. H., Fishburn, P. C., 1996. On the varieties of matrix probabilities in nonarchimedean decision theory. J Mathematical Economics 25, 33-54.

Lindblad, G., 1999. A general no-cloning theorem. Letters in Mathematical Physics 47, 189-196.

Ludwig, G., 1983a. Foundations of Quantum Mechanics I. Springer, New York, translation of Die Grundlagen der Quantenmechanik, Springer 1954.

Ludwig, G., 1985. An axiomatic basis for quantum mechanics, vol. I. Springer, Berlin/Heidelberg/New York.

Mackey, G. W., 1963. The mathematical foundations of quantum mechanics. W. A. Benjamin, New York.

Mielnik, B., 1969. Theory of filters. Commun. Math. Phys. 15, 1-46.

Piron, C., 1976. Foundations of quantum physics. W. A. Benjamin, Reading, MA.

Polchinski, J., 1991. Weinberg's nonlinear quantum mechanics and the einstein-podolskyrosen paradox. Phys. Rev. Lett. 66, 397-400.

Reck, M., Zeilinger, A., Bernstein, H. J., Bertani, P., 1994. Experimental realization of any discrete unitary operator. Phys. Rev. Lett. 73, 58-61.

Rüttiman, G., 1993. Selected topics in the convexity theory of quantum logics, preprint, cited in Beltrametti and Bugajski (1993).

Rüttiman, G. T., 1981. Detectable properties and spectral quantum logics. In: Neumann, H. (Ed.), Interpretations and foundations of quantum mechanics: proceedings of a conference hold in Marburg 28-30 May 1979. Bibliographisches Institut, Zürich.

Schulman, L. S., 1997. Time's Arrows and Quantum Measurement. Cambridge University Press, Cambridge.

Shor, P. W., 1994. Algorithms for quantum computation: discrete logarithms and factoring. Proc. 37th ann. symp. on the foundations of computer science, 56-65.

Shor, P. W., 1997. Polynomial-time algorithms for prime factorization and discrete logarithms on a quantum computer. SIAM J. Comp. , 1484-1509.

Simon, D., 1997. On the power of quantum computation. SIAM J. Comp. 26, 1474-1483.

Valckenborgh, F., 2000. Operational axiomatics and compound systems. In: Coecke, B., Moore, D., Wilce, A. (Eds.), Current research in operational quantum logic. Klüwer, Dordrecht. 
Vinberg, E. B., 1965. The structure of the group of automorphisms of a homogeneous convex cone. Trans. Moscow Math. Soc. 13, 63.

W. Dür, G. Vidal, J. I. C., 2000. Three qubits can be entangled in two inequivalent ways. Physical Review A 62, 062314.

Wallace, D., 2002. Quantum probability and decision theory, revisited. arXiv.org e-print quant-ph/0211014.

Wigner, E. P., 1952. Z. Physik 131, 101.

Wilce, A., 1992. Tensor products in generalized measure theory. Int. J. Theor. Phys. 31, $1915-1928$.

Wilce, A., 1994. A note on partial abelian semigroups, university of Pittsburgh preprint.

Wilce, A., 1998. Perspectivity and congruence in partial abelian semigroups. Mathematica Slovaca 48, 117-135.

Wilce, A., 2000. Test spaces and orthoalgebras. In: Coecke, B., Moore, D., Wilce, A. (Eds.), Current research in operational quantum logic. Klüwer, Dordrecht.

Wootters, W. K., Zurek, W. H., 1982. A single quantum cannot be cloned. Nature 299, 802 .

Zurek, W. H., 1981. Pointer basis of quantum apparatus: Into what mixture does the wavepacket collapse? Phys. Rev. D 24, 1516. 